Mathematisches Forschungsinstitut Oberwolfach

Report No. 9/2009

\title{
Mini-Workshop: Product Systems and Independence in Quantum Dynamics
}

\author{
Organised by \\ B.V. Rajarama Bhat, Bangalore \\ Uwe Franz, Besancon/Sendai \\ Michael Skeide, Campobasso
}

February 15th - February 21st, 2009

\begin{abstract}
Quantum dynamics, both reversible (i.e., closed quantum systems) and irreversible (i.e., open quantum systems), gives rise to product systems of Hilbert spaces or, more generally, of Hilbert modules. When we consider reversible dynamics that dilates an irreversible dynamics, then the product system of the latter is equal to the product system of the former (or is contained in a unique way). Whenever the dynamics is on a proper subalgebra of the algebra of all bounded operators on a Hilbert space, in particular, when the open system is classical (commutative) it is indispensable that we use Hilbert modules.

The product system of a reversible dynamics is intimately related to a filtration of subalgebras that are independent in a state or conditionally independent in a conditional expectation of the reversible system. This has been illustrated in many concrete dilations that have been obtained with the help of quantum stochastic calculus. Here the underlying Fock space or module determines the sort of quantum independence underlying the reversible system.

The mini-workshop brought together experts from quantum dynamics, product systems and quantum independence who have contributed to the general theory or who have studied intriguing examples. As the implications of the tight relationship between product systems and independence had so far been largely neglected, we expect from our mini-workshop a strong innovative impulse to this field.
\end{abstract}

Mathematics Subject Classification (2000): Primary: 46L08,46L53,46L55,81S25,

Secondary: 16W30,46L54,46M05,46M15,60G51,60J25. 


\section{Introduction by the Organisers}

The analysis of dynamical systems, classical or quantum, reversible or irreversible, is a central issue when applying mathematics to natural sciences, economy, sociology, computer and information sciences, and others.

Since William Arveson's fundamental work on product systems of Hilbert spaces (Arveson systems) we know that product systems play an outstanding role in the classification of quantum dynamics. Boris Tsirelson's large classes of examples of spatial product systems of Arveson systems showed that product systems are intimately related to stochastic independence. If classical dynamics should be included, then Hilbert modules are an indispensable tool.

The Mini-Workshop Product Systems and Independence in Quantum Dynamics, concentrated on these connections, with the goal to open a new promising line of research. To achieve this goal we brought together leading experts in the relevant fields. In the first two days, the state of the art was presented in a series of lectures on selected important topics. During last three days more specific aspects were developed in one-hour research talks. The files of some lectures and talks can be found on the web page

$$
\text { http://www . math-inf .uni-greifswald.de/algebra/q-dyn/ }
$$

of the workshop.

Particularly exciting were the open problems sessions. Trying to open a new line of research, it appeared natural to us scheduling two open problem sessions. As a matter of surprise to us, each open problem session took almost two hours. We think it is not possible to underline better the resonance our concept had. It is our wish to share these open problems, and a detailed description can be found in the Open Problems Section.

We wish to thank all participants, whose participation made the workshop a success. We would also like to express our deep gratitude to the MFO, its Director Prof. Dr. Greuel, the Gesellschaft für Mathematische Forschung e.V., and last but surely not least, the staff of the MFO, for offering to us the opportunity to organize this Mini-Workshop.

B. V. Rajarama Bhat, Uwe Franz, Michael Skeide. 


\section{Workshop: Mini-Workshop: Product Systems and Independence in Quantum Dynamics}

\section{Table of Contents}

\section{Introductory Lectures}

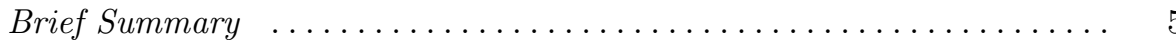

For the organizers: Michael Skeide

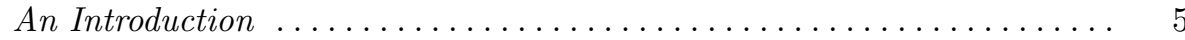

Boris Tsirelson

Brownian Motion and Arveson Systems ................. 13

Volkmar Liebscher

Random Sets and Invariants for Type II Product Systems . . . . . . . . 16

Remus Floricel

CP-Flows and $E_{0}$-Semigroups of Type II $\ldots \ldots \ldots \ldots \ldots \ldots \ldots \ldots$

Michael Schürmann

Classification of Notions of Non-commutative Independence . . . . . . . 19

Claus Köstler

Noncommutative De Finetti Theorems and Noncommutative

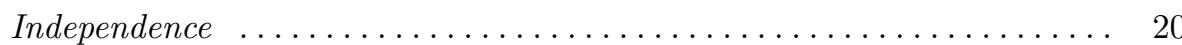

Research Talks

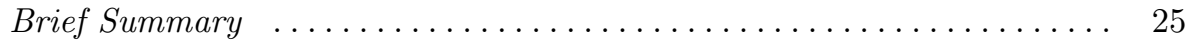

Masaki Izumi

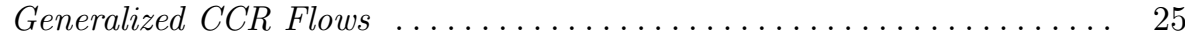

Orr Moshe Shalit

Subproduct Systems and Dilation Theory of CP-Semigroups . . . . . . 27

Claus Köstler

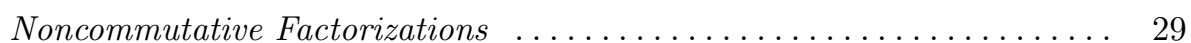

Boris Tsirelson

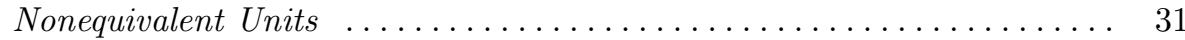

B.V.R. Bhat

Inclusion Systems and Amalgamated Products of Product Systems . . . . 34

Franco Fagnola

Quantum Markov Semigroups and Flows Arising from Form Generators

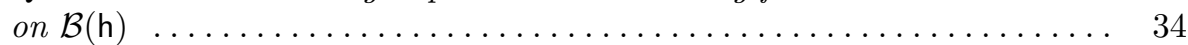

Michael Skeide

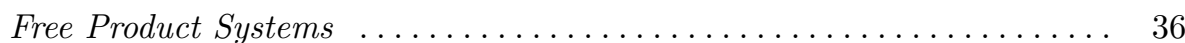


Adam Skalski

Isometric Dilations of Representations of Product Systems of $C^{*}$-Correspondences over $\mathbb{N}_{0}^{k}$

Wilhelm von Waldenfels

An Approach to the Mathematical Theory of Pressure Broadening of Spectral Lines

Michael Schürmann

Transformation of Quantum Lévy Processes

Kalyan B. Sinha

Theory of Integration in Izumi-Srinivasan Generalized CCR Flows . . . . 44

Luigi Accardi

Some Comments on Product Systems and Markov Processes ......... 46

Open Problems ............................ 47 


\section{Abstracts \\ Introductory Lectures - Brief Summary}

The lectures give an up-to-date introduction two some aspects of the workshop which are already well established. Emphasis is on type II Arveson systems (Tsirelson, Liebscher, and Floricel), on axiomatic quantum independence (Schürmann), and on distributional symmetries leading to conditional quantum independence by quantum De Finetti theorems (Köstler). The lectures are preceded by an overall introduction to the concept of the workshop.

\section{An Introduction \\ For the organizers: Michael Skeide}

Idea of the Workshop. All basic (microscopic) laws of nature are reversible, while everyday's experience concerns almost always irreversible phenomena. Understanding irreversible behaviour from first principles is, therefore, a fundamental task. We explain briefly the connection of that task with product systems and independence, claimed in the abstract.

Dynamics, dilations, and modules. An irreversible (quantum) evolution is modelled as a Markov semigroup (that is, a unit-preserving semigroup of completely positive maps) $T=\left(T_{t}\right)_{t \geq 0}$ on a unital $C^{*}$ or von Neumann algebra $\mathcal{B}$. [a] A reversible quantum evolution is modelled as an automorphism group $\alpha=\left(\alpha_{t}\right)$ on some $\mathcal{B}(H)$ ( $H$ a Hilbert space). ${ }^{[\mathrm{b}]}$ Often one restricts attention to a unital $C^{*}$ - or von Neumann subalgebra $\mathcal{A} \subset \mathcal{B}(H)$ that is left invariant by $\alpha_{t}$ for $t \geq 0$. That is, one considers an $E_{0}$-semigroup (that is, a semigroup of unital endomorphisms) $\vartheta=\left(\vartheta_{t}\right)_{t \geq 0}$ on $\mathcal{A}$.

Even if the evolution $\vartheta$ of the algebra $\mathcal{A}$ of observables is reversible, usually not all of $\mathcal{A}$ is accessible to measurement but only a rather small subalgebra $\mathcal{B}$ of $\mathcal{A}$. In particular, if $\mathcal{B}$ consists of macroscopic parameters of the system, then $\mathcal{B}$ is classical, that is, $\mathcal{B}$ is commutative. The evolution $\vartheta$, usually, evolves $\mathcal{B}$ into a subset $\vartheta_{t}(\mathcal{B}) \subset \mathcal{A}$ which is no longer a subset of $\mathcal{B}$. However, since we can only measure the observables in $\mathcal{B}$, what we actually can measure is $\mathfrak{p} \circ \vartheta_{t}(\mathcal{B})$ where $\mathfrak{p}: \mathcal{A} \rightarrow \mathcal{B}$ is a conditional expectation onto $\mathcal{B}$. Clearly, the maps

$$
T_{t}=\mathfrak{p} \circ \vartheta_{t} \uparrow \mathcal{B}
$$

\footnotetext{
[a] The case of a classical Markov semigroup $P=\left(P_{t}\right)_{t>0}$ of transition probabilities on a state space $S$ is included, if we define the positive maps $\left[T_{t}(f)\right](x):=\int_{S} f(y) P_{t}(y, d x)$ on the commutative algebra $L^{\infty}(S)$ of bounded random variables.

${ }^{[b]}$ Wigner's theorem tells us that such an automorphism group is inner, that is, there is a unitary group $u=\left(u_{t}\right)$ in $\mathcal{B}(H)$ such that $\alpha_{t}=u_{t} \bullet u_{t}^{*}$. The unitary group is related to its strong generator, the Hamiltonian of the system, by the Schrödinger equation.
} 
are CP. ${ }^{[c]}$ If the $T_{t}$ form a Markov semigroup $T$ on $\mathcal{B}$, then it is justified to say that we can understand the irreversible evolution of the measurable subsystem $\mathcal{B}$ as projection from the reversible evolution of the whole system. Irreversibility, in that case, is due to the fact that we can never measure all the information about the actual status of the whole system.

In general, starting from a Markov semigroup $T$ on $\mathcal{B}$, one tries to construct a dilation of $T$ that allows to understand $T$ as a projection from a reversible evolution on a bigger system. More precisely, a dilation is a quadruple $(\mathcal{A}, \vartheta, i, \mathfrak{p})$ where $\vartheta$ is an $E_{0}$-semigroup on $\mathcal{A}$, where $i: \mathcal{B} \rightarrow \mathcal{A}$ is an embedding and $\mathfrak{p}: \mathcal{A} \rightarrow \mathcal{B}$ is such that $i \circ \mathfrak{p}$ is a conditional expectation, and where the diagram

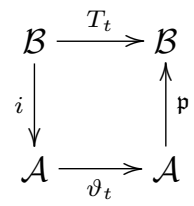

commutes for all $t \geq 0$. A dilation is unital, if $i$ is unital. A dilation is weak if, like in the situation of Footnote $[\mathrm{c}], i(\mathcal{B})$ is a corner of $\mathcal{A}$ and $i \circ \mathfrak{p}=i(\mathbf{1}) \bullet i(\mathbf{1})$.

In this setting, Hilbert modules enter in several ways by Paschke's $\boldsymbol{G N} \boldsymbol{S}$ construction for CP-maps [Pas73]: If $T: \mathcal{A} \rightarrow \mathcal{B}$ is a CP-map between unital $C^{*}$-algebras, then we find a correspondence ${ }^{[\mathrm{d}]} E$ from $\mathcal{A}$ to $\mathcal{B}$ and a vector $\xi \in E$ such that

$$
T(a)=\langle\xi, a \xi\rangle .
$$

Moreover, if $\xi$ generates $E$ as correspondence (that is, if $E=\overline{\operatorname{span}} \mathcal{A} \xi \mathcal{B}$ ), then the pair $(E, \xi)$ is unique up to bilinear unitary equivalence. ${ }^{[\mathrm{e}]}$

Applying the GNS-construction to each $T_{t}$ is at the heart of the construction of product systems for CP-semigroups in [BS00] and, further, a weak dilation. We come back to this, later. Applying, in a dilation of $T$, the GNS-construction to the CP-map $\mathfrak{p}$ we obtain a correspondence $E$ from $\mathcal{A}$ to $\mathcal{B}$ and a unit vector $\xi \in E$ (that is, $\langle\xi, \xi\rangle=\mathbf{1})$ such that $\mathfrak{p}(a)=\langle\xi, a \xi\rangle$ for all $a \in \mathcal{A}$. The left action of $\mathcal{A}$ induces a canonical homomorphism from $\mathcal{A}$ into the adjointable operators $\mathcal{B}^{a}(E)$ on $E$. Experience shows: Firstly, if the canonical homomorphism is not faithful, then normally we may divide out its kernel (the $E_{0}$-semigroup on $\mathcal{A}$ gives rise to

\footnotetext{
[c] If $\mathcal{B}$ contains the unit of $\mathcal{A}$, then the $T_{t}$ are unital, automatically. Also if $\mathcal{B}=p \mathcal{A} p$ is a corner of $\mathcal{A}$ for some projection $p \in \mathcal{A}$ and $\mathfrak{p}=p \bullet p$, then as soon as the $T_{t}$ form a semigroup, $T_{t}$ must be unital.

${ }^{[\mathrm{d}]}$ Correspondence is the fashionable name for Hilbert bimodule. A Hilbert $\mathcal{A}$ - $\mathcal{B}$-bimodule is a (right, of course) Hilbert $\mathcal{B}$-module $E$ with a nondegenerate left action of $\mathcal{A}$. Recall that a correspondence $E$ from $\mathcal{A}$ to $\mathcal{B}$ and a correspondence $F$ from $\mathcal{B}$ to $\mathcal{C}$ have as (internal) tensor product a correspondence $E \odot F$ from $\mathcal{A}$ to $\mathcal{C}$ which is determined uniquely (up to a bilinear unitary) by the requirement that it is generated by elementary tensors $x \odot y$ with inner product $\left\langle x \odot y, x^{\prime} \odot y^{\prime}\right\rangle=\left\langle y,\left\langle x, x^{\prime}\right\rangle y^{\prime}\right\rangle$.

$\left.{ }^{[\mathrm{e}}\right]_{\text {Note that }}$ if $\mathcal{B} \subset \mathcal{B}(G)$ is represented by operators on a Hilbert space, then it is easy to obtain the Stinespring representation [Sti55] of $T$. Indeed, if we put $H:=E \odot G$ (see Footnote [d] for the tensor product), then the induced representation $\rho(a): x \odot g \mapsto a x \odot g$ of $\mathcal{A}$ on $H$ is the Stinespring representation, and $\xi: g \mapsto \xi \odot g$ is an element in $\mathcal{B}(G, H)$ such that $T(a)=\xi^{*} \rho(a) \xi$.
} 
an $E_{0}$-semigroup on the quotient still dilating $T$ ). In other words, we may assume $\mathcal{A} \subset \mathcal{B}^{a}(E)$. Secondly, even if $\mathcal{A} \subsetneq \mathcal{B}^{a}(E)$, then normally the structure (that is, the $E_{0}$-semigroup and, of course, the conditional expectation) extends to all of $\mathcal{B}^{a}(E)$ so that we may assume $\mathcal{A}=\mathcal{B}^{a}(E)$. (We do not know of a single concrete example where it would be known that this does not work.) This is very convenient, because, though sufficiently general for all (known) applications, the algebra $\mathcal{B}^{a}(E)$ preserves much of the simplicity that makes $\mathcal{B}(H)$ more tractable than general von Neumann algebras. On the other hand, we know lots of examples where $\mathcal{B}^{a}(E)$ is contained canonically in some $\mathcal{B}(H)$, and where the $E_{0}$-semigroup on $\mathcal{B}^{a}(E)$ does not extend to $\mathcal{B}(H)$. Also, [Sto72] shows that conditional expectations onto a unital commutative von Neumann subalgebra $\mathcal{B}$ of $\mathcal{B}(H)$ exist if and only if $\mathcal{B}$ is purely atomic. This means, almost never we may hope to dilate a classical irreversible evolution to an $E_{0}$-semigroup on $\mathcal{B}(H)$. But algebras of observables that are measurable simultaneously, are always commutative.

We think that for these and other reasons the use of Hilbert modules in irreversible dynamics is unavoidable. But often the experience in studying Markov semigroups on $\mathcal{B}(G)$ (leading to dilations on $\mathcal{B}(H)$ !) can serve as an optimal preparation for the more general theory.

The objects occurring in dilations, Markov semigroups and $E_{0}$-semigroups, are related in several ways to product systems and independence. Independence itself is intimately related to a large subclass of product systems, the spatial product systems. On the other hand, also the dynamics leading to spatial product systems have intrinsic characterizations as spatial $E_{0}$-semigroups and spatial Markov semigroups.

There are lots of open problems in both the intrinsic classification of product systems - even for Hilbert spaces far from being close to a stable situation, while for modules not more than just started - and classification of dynamics (reversible and irreversible) in terms of their associated product systems. The lectures of Tsirelson (Page 13), of Liebscher (Page 16), and of Floricel (Page 17) deal with spatial Arveson systems (see the next section), and Izumi's talk (Page $25)$ with nonspatial ones. Apart from making progress in these general questions on classification, it is in the scope of the mini-workshop to make progress where there is a relation with independence.

Spatial reversible dynamics and noises. $E_{0}$-semigroups on $\mathcal{B}^{a}(E)$ give rise to product systems. For the case $\mathcal{B}(H)$, in [Arv89a] this marked the beginning of the modern theory of product systems of Hilbert spaces or, for short, Arveson systems; see the monograph [Arv03]. Even in the Hilbert space case the number of new Arveson systems is still increasing every day; see, for instance, [Tsi00a, Tsi00b, Lie03, Pow03, BS05]. The relation between $E_{0}$-semigroups and product systems was generalized to $\mathcal{B}^{a}(E)$ first in [Ske02] (adapting to the module case the approach from [Bha96] to Arveson systems in presence of a unit vector). The most general form is in [Ske04b]. By a product system we mean a family $E^{\odot}=\left(E_{t}\right)_{t \geq 0}$ 
of correspondences over $\mathcal{B}$ that factor in an associative way as

$$
E_{s+t}=E_{s} \odot E_{t}
$$

under tensor product. $E^{\odot}$ is the product system of the $E_{0}$-semigroup $\vartheta$ on $\mathcal{B}^{a}(E)$, if we have identifications $E=E \odot E_{t}$ such that $\vartheta_{t}(a)=a \odot \mathrm{id}_{t} \cdot{ }^{[\mathrm{f}]}$ Depending on the circumstances, there are also technical conditions on both sides, which we do not mention here. Arveson systems classify $E_{0}$-semigroups $\mathcal{B}(H)$ up to cocycle conjugacy (provided $H$ is infinite-dimensional and separable) and for every Arveson system there exists an $E_{0}$-semigroup having that Arveson system; see [Arv89a, Arv90a, Arv89b, Arv90b, Lie03, Ske06a, Arv06]. The (very recent) classification in the module case is not up to cocycle conjugacy but up to stable cocycle conjugacy; see [Ske09a]. The existence results are in [Ske07, Ske09b].

For an $E_{0}$-semigroup on $\mathcal{B}(H)$ with an invariant vector state $\varphi=\langle\Omega, \bullet \Omega\rangle$ (a so-called noise on $\mathcal{B}(H)$ ) the factorization $H=H \otimes E_{t}$ gives rise to a filtration of subalgebras $\mathcal{A}_{t}:=\mathrm{id}_{H} \otimes \mathcal{B}\left(E_{t}\right)$ of $\mathcal{B}(H)$ that is tensor independent in the state $\varphi$. (The increment algebras $\mathcal{A}_{[s, t)}:=\vartheta_{s}\left(\mathcal{A}_{t-s}\right)$ to disjoint intervals commute and the monomials factor in the state.) This is the notion of independence underlying quantum Lévy processes in the sense of [Sch93] that generalizes the notion of classical Lévy processes with with values in groups; see [Fra06, FS99] and also Schürmann's talk (Page 44). Also Tsirelson noises in the sense of [Tsi98, Tsi03] give rise to $E_{0}$-semigroups with invariant vectors states and vice versa; see the lecture of Tsirelson on Page 13. The critical property of an Arveson system $E^{\otimes}$ in order that it be derived from a noise, classical or on $\mathcal{B}(H)$, is that $E^{\otimes}$ is spatial. $E^{\otimes}$ is spatial, if it possesses a unit, that is, a family $u^{\otimes}=\left(u_{t}\right)_{t>0}$ of elements $u_{t} \in E_{t}$ that composes as $u_{s} \otimes u_{t}=u_{s+t}$. The $E_{0}$-semigroups having spatial Arveson system are precisely those that are spatial in the sense of [Pow88]. Not all $E_{0}$-semigroups that are spatial possess an invariant vector state. However, [Pow88] showed that they are always cocycle conjugate to a noise. Recently, in [Ske08] this fact has been used to prove that all spatial Markov semigroups on $\mathcal{B}(G)$ admit a dilation that is a cocycle perturbation of a noise in a very specific way, a so-called Hudson-Parthasarathy dilation. We come back to spatial Markov semigroups, later.

Starting again with an $E_{0}$-semigroup on $\mathcal{B}(H)$ with an invariant vector state $\varphi=\langle\Omega, \bullet \Omega\rangle$, instead of the filtration of subalgebras $\mathcal{A}_{t}:=\mathrm{id}_{H} \otimes \mathcal{B}\left(E_{t}\right)$ and their time shifts, we may use the subalgebras $\widehat{\mathcal{A}}_{t}:=\Omega \Omega^{*} \otimes \mathcal{B}\left(E_{t}\right)$ and their time shifts. This filtration is monotone independent in the sense of [Mur01]. While tensor independence may not be generalized to modules $\left(\operatorname{id}_{E} \odot \mathcal{B}^{a}\left(E_{t}\right)\right.$ does not make sense, in general), monotone independence does generalize. Indeed, if we start with an $E_{0}$-semigroup on $\mathcal{B}^{a}(E)$ with an invariant vector expectation $\varphi=\langle\Omega, \bullet \Omega\rangle$ for a unit vector $\Omega \in E$ (that is, if we start with a noise on $\mathcal{B}^{a}(E)$ in the sense of [Ske06b]), then the filtration of subalgebras $\widehat{\mathcal{A}}_{t}=\left(\Omega \odot \mathrm{id}_{t}\right) \mathcal{B}^{a}\left(E_{t}\right)\left(\Omega^{*} \odot \mathrm{id}_{t}\right)$ and their time shifts is conditionally monotone independent in the vector

\footnotetext{
${ }^{[\mathrm{f}]}$ This is nothing but the representation theory of $\mathcal{B}^{a}(E)$. The most general form is in [MSS06].
} 
expectation $\varphi$ in the sense of [Ske04a]. Also here there is a relation between noises and spatial product systems. Just that the elements of the unit that makes the product system a spatial one, must be central (that is, they must commute with the algebra $\mathcal{B}$ over which the $E_{t}$ are bimodules). Again, spatial Markov semigroups on $\mathcal{B}$ admit Hudson-Parthasarathy dilations; see [Ske09a].

Other types of independence might lead even to new types of product systems. For instance, as indicated in [Ske06b], conditionally free independence in the sense of [Voi95] leads to free product systems (the tensor product of correspondences replaced by the free product). The relation between the tensor product system of such free flows [Fow95, Ske06b] and their free product system is subject to present research, and has been discussed in Skeide's talk, see Page 36.

Voiculescu's free independence [Voi87] is just one, though probably the most fundamental, independence different from monotone or tensor independence. Today there is an impressive (and still increasing) number of quantum independences; see the lectures of Schürmann on Page 19 and of Köstler on Page 20. Next to independence is exchangeability. Köstler explains in his lecture (see Page 20) that such and other distributional symmetries, like in the classical De Finetti theorem, lead to conditional independence.

Many independences have found realizations as noises in quantum dynamics. There are quantum stochastic calculi with respect to these noises that allow to construct dilations of Markov semigroups as cocycle perturbation of the underlying noises. Still today most known examples have been constructed with a calculus for tensor independence based on the fundamental work [HP84]; see the monograph [Par92] and the up-to-date survey [Lin05]. But there are also calculi on other Fock spaces [BSW82, KS92] or modules [GS99, Ske00] or even representation free versions [AFQ92, Kös00]. For all of them a program like the one indicated in the preceding paragraph for the free case still has to be carried out. A detailed study of the underlying product systems promises to provide us with many concrete examples. Stimulating the analysis of how these examples fit into the classification of product systems ([Arv89a, Tsi00a, Lie03, Pow03] for Arveson systems and [LS01, Ske03, BBLS04, Ske06b] for modules) was in the scope of the workshop.

Irreversible dynamics, spatial and not. Clearly, everything that has to do with Markov properties, actually, regards possible notions of conditional independence. Quantum stochastic calculus allowed to construct explicit dilations of Markov semigroups as cocycle perturbations of noises on Fock spaces or Fock modules; see above. These dilations are unital and related to independence in another way through their noises. The construction of a (unique minimal) weak dilation of a Markov semigroup on $\mathcal{B}$ is due to [Bha96] for $\mathcal{B}=\mathcal{B}(G)$ and [Bha99] for a general $C^{*}$-algebra. However, the dilation of [Bha99] is to a $C^{*}$-subalgebra $\mathcal{A}$ of $\mathcal{B}(H)$ and does, in general, not extend to $\mathcal{B}(H)$.

Starting from a CP-semigroup $T$ on $\mathcal{B}$, [BSO0] constructed a product system $E^{\odot}$ and a unit $\xi^{\odot}=\left(\xi_{t}\right)$ such that $T_{t}(b)=\left\langle\xi_{t}, \bullet \xi_{t}\right\rangle$ and such that $E^{\odot}$ is generated

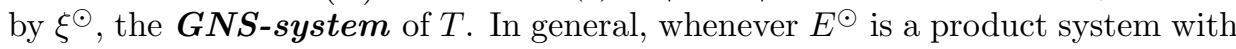
a unital unit $\xi^{\odot}$ (that is, all $\xi_{t}$ are unit vectors) so that $T_{t}(b):=\left\langle\xi_{t}, \bullet \xi_{t}\right\rangle$ defines a 
Markov semigroup $T$, one may construct a weak dilation $\vartheta$ of $T$ to some $\mathcal{B}^{a}(E)$ (in a vector expectation $\langle\xi, \bullet \xi\rangle$ for some unit vector $\xi \in E$ ), which has $E^{\odot}$ as product system.

$\mathcal{B}^{a}(E)$ contains the $C^{*}$-algebra $\mathcal{A}$ of the minimal dilation from [Bha99], and $\vartheta$ restricts to $\mathcal{A}$. If $E^{\odot}$ is the GNS-system of $T$, then $\mathcal{A}$ acts cyclicly on the unit vector $\xi$. (The dilation in [Bha99] extends to $\mathcal{B}^{a}(E) \subset \mathcal{B}(H)$, but not necessarily to $\mathcal{B}(H)$.)

On the other hand, if $E^{\odot}$ can be chosen spatial, then by the classification of $E_{0}$-semigroups, it is stably cocycle conjugate to a noise. This can be used to prove that the GNS-system of a Markov semigroup $T$ can be embedded into a spatial product system if and only if $T$ admits a dilation that is a cocycle perturbation of a noise; see [Ske09a]. This property is equivalent to that $T$ is spatial in a sense generalising naturally the definition of [Arv97] for $\mathcal{B}(G)$.

In [BLS08] it is shown that spatiality of a Markov semigroup on a $C^{*}$-algebra does not imply that the GNS-system is already spatial. But, spatial Markov semigroups on $C^{*}$-algebras are a bit boring, in that they are all uniformly continuous. The von Neumann case allows for strongly continuous spatial Markov semigroups with unbounded generator and all of them have spatial product systems. In fact, we do not know a single nontrivial example of a nonspatial Markov semigroup on $\mathcal{B}(G)$. (Nontrivial means, it is not a nonspatial $E_{0}$-semigroup nor derived from such by trivial tensor product constructions.) On the other hand, we know that the Brownian semigroup and the Ornstein-Uhlenbeck semigroup (on $\mathcal{B}=L^{\infty}(\mathbb{R})$ ) are nonspatial; see [FLS09]. They admit, however, spatial quantum extensions to $\mathcal{B}\left(L^{2}(\mathbb{R})\right)$; see [CFL00]. This raises several natural questions which are discussed in the open problem; see Page 51.

A large class of (spatial) Markov semigroups on $\mathcal{B}(G)$, the largest class we know, have form generators in the sense of [CF98], which resemble the Lindblad form [GKS76, Lin76] of bounded generators; see Fagnola's talk on Page 34. Also here several questions are postponed to the open problems; see Page 51.

Field versions. It is natural to ask for generalizations of the discussion of oneparameter semigroups to more general semigroups or even to fields (that is, without stationarity, therefore, without any semigroup action). There are results in the dilation of two-parameter Markov semigroups in the discrete case by [Sol06] and in the continuous case (under an additional condition that still has to be understood better) by [Sha07]; see the talks by Shalit (Page 27) and by Skalski (Page 39). Accardi describes in his talk (Page 46) a nonstationary construction for classical Markov fields. There are several related open problems; see the Section on Open Problems on Page 47.

Nonspatial dynamics. Nonspatial product systems and the related reversible dynamics fall a bit outside, as far as the direct relation with independence is not given. Nevertheless, we think that at least on two stages there are clear connections. Firstly, once we find (nontrivial) nonspatial Markov semigroups, the dilation problem relates the associated nonspatial product system to conditional 
independence. Secondly, the latest constructions [Tsi00b, BS05] of nonspatial product systems arise by perturbing Fock systems (that is, perturbing not only noises, but even white noises). We think that the question how independence can be perturbed, clearly, should be interesting also for understanding independence. Results like [IS07, Izu07] about the structure of such nonspatial product systems are discussed in Izumi's talk on Page 25.

\section{SOME REFERENCES}

[AFQ92] L. Accardi, F. Fagnola, and J. Quaegebeur, A representation-free quantum stochastic calculus, J. Funct. Anal. 104 (1992), 149-197.

[Arv89a] W. Arveson, Continuous analogues of Fock space, Mem. Amer. Math. Soc., no. 409, American Mathematical Society, 1989.

[Arv89b] - Continuous analogues of Fock space III: Singular states, J. Operator Theory 22 (1989), 165-205.

[Arv90a] _ Continuous analogues of Fock space II: The spectral $C^{*}$-algebra, J. Funct. Anal. 90 (1990), 138-205.

[Arv90b] Continuous analogues of Fock space IV: essential states, Acta Math. 164 (1990), 265-300

[Arv97] , The index of a quantum dynamical semigroup, J. Funct. Anal. 146 (1997), $557-588$

[Arv03] - Noncommutative dynamics and E-semigroups, Monographs in Mathematics, Springer, 2003.

[Arv06] - On the existence of $E_{0}$-semigroups, Infin. Dimens. Anal. Quantum Probab. Relat. Top. 9 (2006), 315-320.

[BBLS04] S.D. Barreto, B.V.R. Bhat, V. Liebscher, and M. Skeide, Type I product systems of Hilbert modules, J. Funct. Anal. 212 (2004), 121-181, (Preprint, Cottbus 2001).

[Bha96] B.V.R. Bhat, An index theory for quantum dynamical semigroups, Trans. Amer. Math. Soc. 348 (1996), 561-583.

[Bha99] _ Minimal dilations of quantum dynamical semigroups to semigroups of endomorphisms of $C^{*}$-algebras, J. Ramanujan Math. Soc. 14 (1999), 109-124.

[BLS08] B.V.R. Bhat, V. Liebscher, and M. Skeide, Subsystems of Fock need not be Fock: Spatial CP-semigroups, arXiv: 0804.2169v1, 2008.

[BS00] B.V.R. Bhat and M. Skeide, Tensor product systems of Hilbert modules and dilations of completely positive semigroups, Infin. Dimens. Anal. Quantum Probab. Relat. Top. 3 (2000), 519-575, (Rome, Volterra-Preprint 1999/0370).

[BS05] B.V.R. Bhat and R. Srinivasan, On product systems arising from sum systems, Infin. Dimens. Anal. Quantum Probab. Relat. Top. 8 (2005), 1-31.

[BSW82] C. Barnett, R.F. Streater, and I.F. Wilde, The Ito-Clifford integral, J. Funct. Anal. 48 (1982), 172-212.

[CE79] E. Christensen and D.E. Evans, Cohomology of operator algebras and quantum dynamical semigroups, J. London Math. Soc. 20 (1979), 358-368.

[CF98] A.M. Chebotarev and F. Fagnola, Sufficient conditions for conservativity of minimal quantum dynamical semigroups, J. Funct. Anal. 153 (1998), 382-404.

[CFL00] F. Cipriani, F. Fagnola, and J.M. Lindsay, Spectral analysis and Feller property for quantum Ornstein-Uhlenbeck semigroups, Commun. Math. Phys. 210 (2000), 85-105.

[FLS09] F. Fagnola, V. Liebscher, and M. Skeide, Product systems of Ornstein-Uhlenbeck processes (tentative title), Preprint, in preparation, 2009.

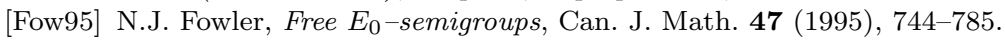

[Fra06] U. Franz, Lévy processes on quantum groups and dual groups, Quantum independent increment processes II (M. Schürmann and U. Franz, eds.), Lect. Notes Math., no. 1866, Springer, 2006, pp. 161-257. 
[FS99] U. Franz and R. Schott, Stochastic processes and operator calculus on quantum groups, Kluwer Academic Publishers, 1999.

[GKS76] V. Gorini, A. Kossakowski, and E.C.G. Sudarshan, Completely positive dynamical semigroups on n-level systems, J. Math. Phys. 17 (1976), 821-825.

[GS99] D. Goswami and K.B. Sinha, Hilbert modules and stochastic dilation of a quantum dynamical semigroup on a von Neumann algebra, Commun. Math. Phys. 205 (1999), $377-403$.

[HP84] R.L. Hudson and K.R. Parthasarathy, Quantum Ito's formula and stochastic evolutions, Commun. Math. Phys. 93 (1984), 301-323.

[IS07] M. Izumi and R. Srinivasan, Generalized CCR flows, Preprint, arXiv: 0705.3280v1, 2007.

[Izu07] M. Izumi, Every sum system is divisible, Preprint, arXiv: 0708.1591v1, 2007.

[Kös00] C. Köstler, Quanten-Markoff-Prozesse und Quanten-Brownsche Bewegungen, Ph.D. thesis, Stuttgart, 2000

[KS92] B. Kümmerer and R. Speicher, Stochastic integration on the Cuntz algebra $O_{\infty}$, J. Funct. Anal. 103 (1992), 372-408.

[Lie03] V. Liebscher, Random sets and invariants for (type II) continuous tensor product systems of Hilbert spaces, Preprint, arXiv: math.PR/0306365, 2003, To appear in Mem. Amer. Math. Soc.

[Lin76] G. Lindblad, On the generators of quantum dynamical semigroups, Commun. Math. Phys. 48 (1976), 119-130.

[Lin05] J.M. Lindsay, Quantum stochastic analysis — an introduction, Quantum independent increment processes I (M. Schürmann and U. Franz, eds.), Lect. Notes Math., no. 1865, Springer, 2005, pp. 181-271.

[LS01] V. Liebscher and M. Skeide, Units for the time ordered Fock module, Infin. Dimens. Anal. Quantum Probab. Relat. Top. 4 (2001), 545-551, (Rome, Volterra-Preprint 2000/0411).

[MSS06] P.S. Muhly, M. Skeide, and B. Solel, Representations of $\mathcal{B}^{a}(E)$, Infin. Dimens. Anal. Quantum Probab. Relat. Top. 9 (2006), 47-66, (arXiv: math.OA/0410607).

[Mur01] N. Muraki, Monotonic independence, monotonic central limit theorem and monotonic law of small numbers, Infin. Dimens. Anal. Quantum Probab. Relat. Top. 4 (2001), $39-58$.

[Par92] K.R. Parthasarathy, An introduction to quantum stochastic calculus, Birkhäuser, 1992.

[Pas73] W.L. Paschke, Inner product modules over $B^{*}$-algebras, Trans. Amer. Math. Soc. 182 (1973), 443-468.

[Pow88] R.T. Powers, An index theory for semigroups $*$-endomorphisms of $\mathfrak{B}(\mathfrak{H})$, Can. Jour. Math. 40 (1988), 86-114.

[Pow03] _ Continuous spatial semigroups of completely positive maps of $\mathfrak{B}(\mathfrak{H})$, New York J. Math. 9 (2003), 165-269, Available at http://nyjm.albany.edu:8000/j/2003/9-13.html.

[Sch93] M. Schürmann, White noise on bialgebras, Lect. Notes Math., no. 1544, Springer, 1993.

[Sha07] O.M. Shalit, $E_{0}$-dilation of strongly commuting $C_{0}$-semigroups, Preprint, arXiv: 0707.1760v2 [math.OA], 2007, To appear in J. Funct. Anal.

[Ske00] M. Skeide, Quantum stochastic calculus on full Fock modules, J. Funct. Anal. 173 (2000), 401-452, (Rome, Volterra-Preprint 1999/0374).

[Ske02] _ Dilations, product systems and weak dilations, Math. Notes 71 (2002), 914-923.

[Ske03] Dilation theory and continuous tensor product systems of Hilbert modules, Quantum Probability and Infinite Dimensional Analysis (W. Freudenberg, ed.), Quantum Probability and White Noise Analysis, no. XV, World Scientific, 2003, Preprint, Cottbus 2001, pp. 215-242.

[Ske04a] _ Independence and product systems, Recent developments in stochastic analysis and related topics (S. Albeverio, Z.-M. Ma, and M. Röckner, eds.), World Scientific, 2004, (arXiv: math.OA/0308245), pp. 420-438. 
[Ske04b] _ Unit vectors, Morita equivalence and endomorphisms, Preprint, arXiv: math.OA/0412231v5 (Version 5), 2004, To appear in Publ. Res. Inst. Math. Sci. 45 (2) 2009 .

[Ske06a] — Existence of $E_{0}$-semigroups for Arveson systems: Making two proofs into one, Infin. Dimens. Anal. Quantum Probab. Relat. Top. 9 (2006), 373-378, (arXiv: math.OA/0605480)

[Ske06b] _ The index of (white) noises and their product systems, Infin. Dimens. Anal. Quantum Probab. Relat. Top. 9 (2006), 617-655, (Rome, Volterra-Preprint 2001/0458, arXiv: math.OA/0601228).

[Ske07] _ E E E-semigroups for continuous product systems, Infin. Dimens. Anal. Quantum Probab. Relat. Top. 10 (2007), 381-395, (arXiv: math.OA/0607132).

[Ske08] _ Spatial Markov semigroups admit Hudson-Parthasarathy dilations, Preprint, arXiv: $0809.3538 \mathrm{v} 1,2008$.

[Ske09a] _ Classification of $E_{0}$-semigroups by product systems, Preprint, arXiv: $0901.1798 v 1,2009$.

[Ske09b] _ Dilations of product systems and commutants of von Neumann modules, Preprint, in preparation, 2009.

[Sol06] B. Solel, Representations of product systems over semigroups and dilations of commuting CP maps, J. Funct. Anal. 235 (2006), 593-618, (arXiv: math.OA/0502423).

[Sti55] W.F. Stinespring, Positive functions on $C^{*}$-algebras, Proc. Amer. Math. Soc. 6 (1955), 211-216

[Sto72] E. Stormer, On projection maps of von Neumann algebras, Math. Scand. 30 (1972), 46-50.

[Tsi98] B. Tsirelson, Unitary Brownian motions are linearizable, Preprint, arXiv: math.PR/9806112, 1998.

[Tsi00a] _ From random sets to continuous tensor products: answers to three questions of W. Arveson, Preprint, arXiv: math.FA/0001070, 2000.

[Tsi00b] _ From slightly coloured noises to unitless product systems, Preprint, arXiv: math.FA/0006165, 2000

[Tsi03] _ _ Scaling limit, noise, stability, Preprint, arXiv: math.PR/0301237, 2003.

[Voi87] D. Voiculescu, Dual algebraic structures on operator algebras related to free products, J. Operator Theory 17 (1987), 85-98.

[Voi95] Operations on certain non-commutative operator-valued random variables, Astérisque 232 (1995), 243-275.

\section{Brownian Motion and Arveson Systems BORIS TSIRELSON}

Elaborate constructions (especially, counterexamples) in a Hilbert space often use a coordinate system (orthonormal basis). In other words, the sequence space $l_{2}$ is used rather than an abstract Hilbert space. An Arveson system consists of Hilbert spaces, but we cannot choose their bases without sacrificing the given tensor product structure. Instead, we can choose maximal commutative operator algebras, which leads to the probabilistic approach. Especially, the white noise (or Brownian motion) will be used rather than an abstract type $I_{1}$ Arveson system.

I do not reproduce here the definition of an Arveson system [1, 3.1.1], since we only need the special case

$$
H_{t}=L_{2}\left(\Omega, \mathcal{F}_{0, t}, P\right)
$$


corresponding to a noise, for now, to the white noise generated by the increments of the one-dimensional Brownian motion $\left(B_{t}\right)_{-\infty<t<\infty}, B_{t}: \Omega \rightarrow \mathbb{R}$. The relation $H_{s} \otimes H_{t}=H_{s+t}$ for Hilbert spaces (or rather a unitary operator $H_{s} \otimes H_{t} \rightarrow H_{s+t}$ ) emerges naturally from the similar relation for probability spaces.

We specialize the definition of a unit $[1,3.6 .1]$ to systems of the form (1).

Definition 1. A unit (of the system (1)) is a family $\left(u_{t}\right)_{t>0}$ of non-zero vectors $u_{t} \in H_{t}=L_{2}\left(\mathcal{F}_{0, t}\right) \subset L_{2}(\mathcal{F})$ such that $t \mapsto u_{t}$ is a Borel measurable map $(0, \infty) \rightarrow$ $L_{2}(\mathcal{F})$, and

$$
u_{s} u_{t}=u_{s+t} \text { for all } s, t>0 .
$$

(In other words, the given unitary operator $H_{s} \otimes H_{t} \rightarrow H_{s+t}$ maps $u_{s} \otimes u_{t}$ to $u_{s+t}$.) The unit is normalized, if $\left\|u_{t}\right\|=1$ for all $t$. (In general, $\left\|u_{t}\right\|=\exp (c t)$ for some $c \in \mathbb{R}$.)

Here is the general form of a unit in our $\left(H_{t}\right)_{t}$ :

$$
u_{t}=\exp \left(z B_{t}+z_{1} t\right) ; \quad z, z_{1} \in \mathbb{C} ;
$$

it is normalized iff $(\operatorname{Re} z)^{2}+\operatorname{Re} z_{1}=0$. The units generate $\left(H_{t}\right)_{t}$ in the following sense: for every $t>0, H_{t}$ is the closed linear span of vectors of the form $\left(u_{1}\right)_{\frac{t}{n}}\left(u_{2}\right)_{\frac{t}{n}} \ldots\left(u_{n}\right)_{\frac{t}{n}}$, where $u_{1}, \ldots, u_{n}$ are units, $n=1,2, \ldots$ Indeed, $L_{2}\left(\mathcal{F}_{0, t}\right)$ is spanned by random variables of the form $\exp \left(\mathrm{i} \int_{0}^{t} f(s) \mathrm{d} B_{s}\right)$ where $f$ runs over step functions $(0, t) \rightarrow \mathbb{R}$ constant on $\left(0, \frac{1}{n} t\right), \ldots,\left(\frac{n-1}{n} t, t\right)$. (1).

We specialize two notions, 'type $I$ ' and 'automorphism', to systems of the form

Definition 2. A system of the form (1) is of type $I$, if it is generated by its units.

We see that our $\left(H_{t}\right)_{t}$ is of type $I$.

Definition 3. An automorphism (of the system (1)) is a family $\left(\Theta_{t}\right)_{t>0}$ of unitary operators $\Theta_{t}: H_{t} \rightarrow H_{t}$ such that $\Theta_{s+t}(X Y)=\left(\Theta_{s} X\right)\left(\Theta_{t} Y\right)$ for all $X \in H_{s}$, $Y \in H_{t}, s>0, t>0$, and the function $t \mapsto\left\langle\Theta_{t} X_{t}, Y_{t}\right\rangle$ is Borel measurable whenever $t \mapsto X_{t}$ and $t \mapsto Y_{t}$ are Borel measurable maps $(0, \infty) \rightarrow L_{2}(\mathcal{F})$ such that $X_{t}, Y_{t} \in L_{2}\left(\mathcal{F}_{0, t}\right) \subset L_{2}(\mathcal{F})$.

Basically, $\Theta_{s} \otimes \Theta_{t}=\Theta_{s+t}$. The group $G$ of all automorphisms is called the gauge group. Clearly, $G$ acts on the set of normalized units, $\left(u_{t}\right)_{t} \mapsto\left(\Theta_{t} u_{t}\right)_{t}$.

Normalized units $\left(u_{t}\right)_{t}$ and $\left(\mathrm{e}^{\mathrm{i} \lambda t} u_{t}\right)_{t}$ will be called equilavent. The gauge group $G$ acts on the set of all equivalence classes of normalized units.

We turn to the gauge group $G$ of our type $I$ system $\left(H_{t}\right)_{t}$. Equivalence classes of normalized units of $\left(H_{t}\right)_{t}$ are parametrized by numbers $z \in \mathbb{C}$, since each class contains exactly one unit of the form

$$
u_{t}=\exp \left(z B_{t}-(\operatorname{Re} z)^{2} t\right) .
$$

The scalar product corresponds to the distance:

$$
\left|\left\langle u_{t}^{(1)}, u_{t}^{(2)}\right\rangle\right|=\exp \left(-\frac{1}{2}\left|z_{1}-z_{2}\right|^{2} t\right)
$$


for $u_{t}^{(k)}=\exp \left(z_{k} B_{t}-\left(\operatorname{Re} z_{k}\right)^{2} t\right), k=1,2$. The action of $G$ on equivalence classes boils down to its action on $\mathbb{C}$ by isometries. The orientation of $\mathbb{C}$ is preserved, since

$$
\frac{\left\langle u_{t}^{(1)}, u_{t}^{(2)}\right\rangle\left\langle u_{t}^{(2)}, u_{t}^{(3)}\right\rangle\left\langle u_{t}^{(3)}, u_{t}^{(1)}\right\rangle}{\left|\left\langle u_{t}^{(1)}, u_{t}^{(2)}\right\rangle\left\langle u_{t}^{(2)}, u_{t}^{(3)}\right\rangle\left\langle u_{t}^{(3)}, u_{t}^{(1)}\right\rangle\right|}=\exp \left(\mathrm{i} t S\left(z_{1}, z_{2}, z_{3}\right)\right),
$$

where $S\left(z_{1}, z_{2}, z_{3}\right)=\Im\left(\left(z_{2}-z_{1}\right) \overline{\left(z_{3}-z_{1}\right)}\right)$ is twice the signed area of the triangle. So, $G$ acts on $\mathbb{C}$ by motions (see $[1,3.8 .4]$ ).

Shifts of $\mathbb{C}$ along the imaginary axis, $z \mapsto z+\mathrm{i} \lambda$ (for $\lambda \in \mathbb{R}$ ) emerge from automorphisms

$$
\Theta_{t}=\Theta_{t}^{\text {shift }(\mathrm{i} \lambda)}=\exp \left(\mathrm{i} \lambda B_{t}\right)
$$

here the random variable $\exp \left(\mathrm{i} \lambda B_{t}\right) \in L_{\infty}\left(\mathcal{F}_{0, t}\right)$ is treated as the multiplication operator, $X \mapsto X \exp \left(\mathrm{i} \lambda B_{t}\right)$ for $X \in L_{2}\left(\mathcal{F}_{0, t}\right)$.

Shifts of $\mathbb{C}$ along the real axis, $z \mapsto z+\lambda$ (for $\lambda \in \mathbb{R}$ ) emerge from less evident automorphisms

$$
\Theta_{t}^{\text {shift }(\lambda)} X=D_{t}^{1 / 2} \cdot\left(X \circ \theta_{t}^{\lambda}\right) ;
$$

here $\theta_{t}^{\lambda}: C[0, t] \rightarrow C[0, t]$ is the drift transformation $\left(\theta_{t}^{\lambda} b\right)(s)=b(s)-2 \lambda s$ (for $s \in[0, t]), D_{t}$ is the Radon-Nikodym derivative of the Wiener measure shifted by $\theta_{t}^{\lambda}$ w.r.t. the Wiener measure itself,

$$
D_{t}=\exp \left(2 \lambda B_{t}-2 \lambda^{2} t\right),
$$

and $X \in L_{2}\left(\mathcal{F}_{0, t}\right)$ is treated as a function on $C[0, t]$ (measurable w.r.t. the Wiener measure). Thus,

$$
\left(\Theta_{t}^{\operatorname{shift}(\lambda)} X\right)(b)=\exp \left(\lambda b(t)-\lambda^{2} t\right) X\left(\theta_{t}^{\lambda} b\right) .
$$

By the way, these two one-parameter subgroups of $G$ satisfy Weyl relations

$$
\Theta_{t}^{\text {shift }(\lambda)} \Theta_{t}^{\text {shift }(\mathrm{i} \mu)}=\mathrm{e}^{-2 \mathrm{i} \lambda \mu t} \Theta_{t}^{\text {shift }(\mathrm{i} \mu)} \Theta_{t}^{\text {shift }(\lambda)} ;
$$

that is, $\Theta^{\text {shift }(\lambda)} \Theta^{\text {shift }(\mathrm{i} \mu)}=\Theta^{\operatorname{trivial}(-2 \lambda \mu)} \Theta^{\operatorname{shift}(\mathrm{i} \mu)} \Theta^{\operatorname{shift}(\lambda)}$.

Rotations of $\mathbb{C}$ around the origin, $z \mapsto \mathrm{e}^{\mathrm{i} \lambda} z$ (for $\lambda \in \mathbb{R}$ ) emerge from automorphisms $\Theta^{\text {rotat }(\lambda)}$. These will not be used, but are briefly described anyway. They preserve Wiener chaos spaces $H_{n}$,

$$
\Theta_{t}^{\operatorname{rotat}(\lambda)} X=\mathrm{e}^{\mathrm{i} n \lambda} X \quad \text { for } X \in H_{n} \cap L_{2}\left(\mathcal{F}_{0, t}\right) ;
$$

the $n$-th chaos space $H_{n} \subset L_{2}(\mathcal{F})$ consists of stochastic integrals

$$
X=\int \cdots \int_{-\infty<s_{1}<\cdots<s_{n}<\infty} f\left(s_{1}, \ldots, s_{n}\right) \mathrm{d} B_{s_{1}} \ldots \mathrm{d} B_{s_{n}}
$$

where $f \in L_{2}\left(\mathbb{R}^{n}\right)$ (or rather, the relevant part of $\mathbb{R}^{n}$ ). One may say that $\Theta^{\operatorname{rotat}(\lambda)}$ just multiplies each $\mathrm{d} B_{s}$ by $\mathrm{e}^{\mathrm{i} \lambda}$.

Combining shifts and rotations we get all motions of $\mathbb{C}$. Accordingly, all automorphisms of $\left(H_{t}\right)_{t}$ are combinations of $\Theta^{\text {shift }(\mathrm{i} \lambda)}, \Theta^{\text {shift }(\lambda)}, \Theta^{\text {rotat }(\lambda)}$ and $\Theta^{\text {trivial }(\lambda)}$. More generally, the $N$-dimensional Brownian motion leads to the (unique up to isomorphism) Arveson system of type $I_{N}$ and motions of $\mathbb{C}^{N}$. We need $N=1$ only; $\left(H_{t}\right)_{t}$ is the Arveson system of type $I_{1}$. 
See also Sect. 1 of [2].

\section{REFERENCES}

[1] W. Arveson, Noncommutative dynamics and E-semigroups, Springer, New York, 2003.

[2] B. Tsirelson, On automorphisms of type II Arveson systems (probabilistic approach), New York Journal of Mathematics 14 (2008), 539-576.

\section{Random Sets and Invariants for Type II Product Systems VOLKMAR LIEBSCHER}

This lecture summarises some results from [1], [3]. Any closed set $Z \subseteq[0,1]$ is characterised by the values $X_{s, t}=\chi_{\left\{Z^{\prime}: Z^{\prime} \cap[s, t]=\emptyset\right\}}(Z)$ for $0 \leq s<t \leq 1$. For random closed sets, $X_{s, t}$ are random variables fulfilling the relation

$$
X_{r, s} X_{s, t}=X_{r, t} .
$$

The distribution of these random variables determines the distribution of a random closed set $Z$. This is manifested by the fact that a probability measure $\mu$ is uniquely determined by the function $c_{\mu}:\{G \subseteq[0,1],: G$ open $\} \mapsto[0,1], c_{\mu}(G)=\mu(\{Z$ : $Z \cap G \neq \emptyset\}$ ), which has to be a Choquet capacity of infinite order:

$$
\sum_{S \subseteq\{1, \ldots, n\}}(-1)^{n-\# S} c_{\mu}\left(G \cup \bigcup_{i \in S} G_{i}\right) \geq 0
$$

for all $G, G_{1}, \ldots, G_{n}$.

Let $\left(\mathcal{E}_{t}\right)_{t \geq 0}$ be a product system of Hilbert spaces, i.e. $\mathcal{E}_{s+t} \cong \mathcal{E}_{s} \otimes \mathcal{E}_{t}$ in a consistent and measurable manner. To any product subsystem $\left(\mathcal{F}_{t}\right)_{t \geq 0}$ one can associate the family $\left(P_{s, t}^{\mathcal{F}}\right)_{0 \leq s<t \leq 1} \in \mathcal{B}\left(\mathcal{E}_{1}\right)$,

$$
\mathrm{P}_{s, t}^{\mathcal{F}}=1_{\mathcal{E}_{s}} \otimes \operatorname{Pr}_{\mathcal{F}_{t-s}} \otimes 1_{\mathcal{E}_{1-t}}
$$

These commuting projection fulfil the similar relation

$$
\mathrm{P}_{r, s}^{\mathcal{F}} \mathrm{P}_{s, t}^{\mathcal{F}}=\mathrm{P}_{r, t}^{\mathcal{F}} \text {. }
$$

The reported result is that we can fix in a sense a distribution of these (quantum stochastic) random variables and even a distribution of a random closed set. Namely, for all normal states $\eta$ on $\mathcal{B}\left(\mathcal{E}_{1}\right)$ there is a unique probability measure $\mu_{\eta}$ on $\{Z \subseteq[0,1]: Z$ closed $\}$ with

$$
\int X_{s_{1}, t_{1}} \cdots X_{s_{k}, t_{k}} \mathrm{~d} \mu_{\eta}=\eta\left(\mathrm{P}_{s_{1}, t_{1}}^{\mathcal{F}} \cdots \mathrm{P}_{s_{k}, t_{k}}^{\mathcal{F}}\right)
$$

for all $0 \leq s_{i}<t_{i} \leq 1, i=1, \ldots, k$. Further, if $\omega$ is faithful, there is a unique isomorphism $j_{\omega}: L^{\infty}\left(\mu_{\omega}\right) \mapsto\left\{P_{s, t}^{\mathcal{F}}: 0 \leq s<t \leq 1\right\}^{\prime \prime}$ extending

$$
j_{\omega}\left(X_{s, t}\right)=\mathrm{P}_{s, t}^{\mathcal{F}}
$$

for all $0 \leq s<t \leq 1$.

If $\mathcal{F}$ is the product system generated by all units $\xi$ of $\mathcal{E}$, i.e. factorising vectors

$$
\xi_{s+t}=\xi_{s} \otimes \xi_{t},
$$


then $\left\{\mu_{\omega}: \omega\right.$ faithful normal state on $\left.\mathcal{B}\left(\mathcal{E}_{1}\right)\right\}$ is an invariant of the product system. The range of this invariant can be fully characterised.

\section{REFERENCES}

[1] V.Liebscher Random set and invariants for (Type II) Product systems of Hilbert spaces to appear in Mem.AMS. 2009

[2] B. Tsirelson From random sets to continuous tensor products: answers to three questions of W. Arveson preprint math/0001070 2000

[3] B. Tsirelson, Non-Isomorphic product systems, preprint math.FA/0210457, 2003.

\section{$C P$-Flows and $E_{0}$-Semigroups of Type II REMus FlORICEL}

Our purpose in this expository note is to give an account of R.T. Powers' approach to classification of $E_{0}$-semigroups of type II ([4], [3], [1]).

As shown by Bhat [2], $E_{0}$-semigroups can be obtained as dilations of semigroups of unital completely positive maps ( $C P_{0}$-semigroups). More precisely, if $\alpha$ is a $C P_{0}$-semigroup of $\mathfrak{B}(H)$, then there is a Hilbert space $H_{1}$, an $E_{0}$-semigroup $\alpha^{d}$ of $\mathfrak{B}\left(H_{1}\right)$, and an isometry $W: H \rightarrow H_{1}$ such that $\alpha_{t}=\operatorname{Ad}\left[W^{*}\right] \circ \alpha_{t}^{d} \circ \operatorname{Ad}[W]$, $t \geq 0$. Moreover, if the projection $E=W W^{*}$ is minimal, i.e.,

$$
\overline{\mathrm{sp}}\left\{\prod_{i=1}^{n} \alpha_{t_{i}}^{d}\left(E A_{i} E\right) W \xi \mid \xi \in H, A_{i} \in \mathfrak{B}(H), t_{i} \geq 0, n \in \mathbb{N}\right\}=H_{1},
$$

then $\alpha^{d}$ is unique up to conjugacy. The $E_{0}$-semigroup $\alpha^{d}$ is called the minimal dilation of $\alpha$.

Minimal dilations of $C P_{0}$-semigroups can be described and classified up to cocycle conjugacy in terms of the associated corners ([3]). Recall that if $\alpha$ and $\beta$ are $C P_{0}$-semigroups of $\mathfrak{B}\left(H_{1}\right)$ and $\mathfrak{B}\left(H_{2}\right)$, then a corner from $\alpha$ to $\beta$ is a family $\gamma=\left\{\gamma_{t} \mid t \geq 0\right\}$ of mappings $\gamma_{t}: \mathfrak{B}\left(H_{1}, H_{2}\right) \rightarrow \mathfrak{B}\left(H_{1}, H_{2}\right)$ such that $\Theta=\left\{\Theta_{t} \mid t \geq 0\right\}$ is a $C P$-semigroup of $\mathfrak{B}\left(H_{1} \oplus H_{2}\right)$, where

$$
\Theta_{t}\left(\left[\begin{array}{ll}
X_{11} & X_{12} \\
X_{21} & X_{22}
\end{array}\right]\right)=\left(\left[\begin{array}{cc}
\alpha_{t}\left(X_{11}\right) & \gamma_{t}\left(X_{12}\right) \\
\gamma_{t}^{*}\left(X_{21}\right) & \beta_{t}\left(X_{22}\right)
\end{array}\right]\right)
$$

for all $t \geq 0, X_{i j} \in \mathfrak{B}\left(H_{i}, H_{j}\right)$. $\gamma$ is said to be a hyper-maximal corner, if for every subordinate $C P$-semigroup $\Theta^{\prime} \leq \Theta$ of the form

$$
\Theta_{t}^{\prime}\left(\left[\begin{array}{ll}
X_{11} & X_{12} \\
X_{21} & X_{22}
\end{array}\right]\right)=\left(\left[\begin{array}{cc}
\alpha_{t}^{\prime}\left(X_{11}\right) & \gamma_{t}\left(X_{12}\right) \\
\gamma_{t}^{*}\left(X_{21}\right) & \beta_{t}^{\prime}\left(X_{22}\right)
\end{array}\right]\right)
$$

one has $\alpha^{\prime}=\alpha$ and $\beta^{\prime}=\beta$. The minimal dilations $\alpha^{d}$ and $\beta^{d}$ of $\alpha$ and $\beta$ are then cocycle conjugate if and only if there is a hyper-maximal corner $\gamma$ from $\alpha$ to $\beta$ $([3])$.

The simplest objects that can be dilated to spatial $E_{0}$-semigroups are the $C P$-flows ([4]): if $K$ is a Hilbert space, $H=K \otimes L^{2}\left(\mathbb{R}_{+}\right)=L^{2}\left(\mathbb{R}_{+}, K\right)$, and $U=\left\{U_{t}\right\}_{t \geq 0}$ is the right translation semigroup of $H$, then a $C P$-flow over $K$ is a $C P$-semigroup $\alpha$ of $\mathfrak{B}(H)$ which is intertwined by $U$, i.e., $\alpha_{t}(A) U_{t}=U_{t} A$, for all 
$A \in \mathfrak{B}(H)$ and $t \geq 0$.

As shown by Powers ([4]), the problem of constructing $C P$-flows is equivalent to constructing $q$-positive boundary weight maps, objects that are defined as follows. Consider the contraction $\Lambda: \mathfrak{B}(K) \rightarrow \mathfrak{B}(H),(\Lambda(A) f)(x)=e^{-x} A f(x), A \in \mathfrak{B}(K)$, $f \in H, x \geq 0$, and denote $\Lambda=\Lambda(I)$. The boundary algebra $\mathcal{A}(H)$ of $\mathfrak{B}(H)$ is the algebra of all operators of the form $A=(I-\Lambda)^{\frac{1}{2}} B(I-\Lambda)^{\frac{1}{2}}, B \in \mathfrak{B}(H)$. We say that $\omega$ is a boundary weight on $\mathcal{A}(H)$ (write $\omega \in \mathcal{A}(H)_{*}$ ), if $\omega$ is a linear functional on $\mathcal{A}(H)$ (a possibly unbounded weight on $\mathfrak{B}(H)$ ), and there is $\mu \in \mathfrak{B}(H)_{*}$ such that $\omega\left((I-\Lambda)^{\frac{1}{2}} A(I-\Lambda)^{\frac{1}{2}}\right)=\mu(A), \quad A \in \mathfrak{B}(H)$. A linear completely bounded map $\mathfrak{B}(K)_{*} \ni \rho \longmapsto \omega(\rho) \in \mathcal{A}(H)_{*}$ is said to be a $q$-positive boundary weight map if the mappings

$$
\widehat{\pi}_{t}:=\omega_{t} \circ\left(I+\widehat{\Lambda} \omega_{t}\right)^{-1}
$$

are completely positive. Here $\omega_{t} \in \mathfrak{B}(H)_{*}$ is the truncated boundary weight $\omega_{t}(A)=\omega\left(U_{t} U_{t}^{*} A U_{t} U_{t}^{*}\right), A \in \mathfrak{B}(H)$, and the symbol "^" denotes the extension to the predual.

The family $\pi^{\#}=\left\{\pi_{t}^{\#} \mid t>0\right\}$ of completely positive contractions $\pi_{t}^{\#}=\pi_{t}$ of $\mathfrak{B}(H)$ into $\mathfrak{B}(K)$, called the generalized boundary representation of the $q$-positive boundary weight map $\rho \longmapsto \omega(\rho)$, provides information about the structure of the minimal dilation $\alpha^{d}$ of a $C P$-flow $\alpha$ over $K$ associated to $\rho \longmapsto \omega(\rho)$ ([4], [3]). Indeed, the index of $\alpha^{d}$ is is the rank of the normal spine $\pi_{0}^{\#}=\lim _{t \rightarrow 0^{+}} \pi_{t}^{\#}$ of the generalized boundary representation $\pi^{\#}=\left\{\pi_{t}^{\#} \mid t>0\right\}$ of $\rho \longmapsto \omega(\rho)$, and $\alpha^{d}$ is of type I if and only if $\alpha$ is the minimal $C P$ - flow derived from $\pi_{0}^{\#}$.

If the Hilbert space $K$ is 1-dimensional, then any $q$-positive boundary weight map is specified by a single boundary weight $\omega$ on the boundary algebra $\mathcal{A}(H)$. Moreover, if $\omega$ is infinite, then the minimal dilation $\alpha^{d}$ of the $C P$-flow $\alpha$ associated to $\omega$ is of type $\mathrm{II}_{0}$, and the corners from $\alpha$ to $\alpha$ can be described explicitly ([1], $[4])$.

\section{REFERENCES}

[1] Alevras, Alexis; Powers, Robert T. Price, Geoffrey L. Cocycles for one-parameter flows of $B(H)$. J. Funct. Anal. 230 (2006), no. 1, 1-64.

[2] Bhat, B. V. Rajarama. An index theory for quantum dynamical semigroups. Trans. Amer. Math. Soc. 348 (1996), no. 2, 561-583.

[3] Powers, Robert T. Construction of $E_{0}$-semigroups of $B(H)$ from $C P$-flows. Advances in quantum dynamics (South Hadley, MA, 2002), 57-97, Contemp. Math., 335, Amer. Math. Soc., Providence, RI, 2003.

[4] Powers, Robert T. Continuous spatial semigroups of completely positive maps of $B(H)$. New York J. Math. 9 (2003), 165-269. 


\section{Classification of Notions of Non-commutative Independence Michael SchÜRMANN}

A natural product for linear functionals on algebras assigns to each pair of linear functionals $\left(\varphi_{1}, \varphi_{2}\right), \varphi_{i}: \mathcal{A}_{i} \rightarrow \mathbb{C}, i=1,2$, a linear functional $\varphi_{1} \bullet \varphi_{2}$ on the free product $\mathcal{A}_{1} \sqcup \mathcal{A}_{2}$ of the algebras $\mathcal{A}_{i}$ such that

$$
\begin{aligned}
\left(\varphi_{1} \bullet \varphi_{2}\right) \circ \iota_{1} & =\varphi_{1} \\
\left(\varphi_{1} \bullet \varphi_{2}\right) \circ \iota_{2} & =\varphi_{2} \\
\left(\varphi_{1} \bullet \varphi_{2}\right) \bullet \varphi_{3} & =\varphi_{1} \bullet\left(\varphi_{2} \bullet \varphi_{3}\right) \\
\left(\varphi_{1} \circ j_{1}\right) \bullet\left(\varphi_{2} \circ j_{2}\right) & =\left(\varphi_{1} \bullet \varphi_{2}\right) \circ\left(j_{1} \sqcup j_{2}\right)
\end{aligned}
$$

where $\iota_{i}$ are the canonical embeddings of $\mathcal{A}_{i}$ into $\mathcal{A}_{1} \sqcup \mathcal{A}_{2}$, and $j_{i}: \mathcal{B}_{i} \rightarrow \mathcal{A}_{i}$ are *-algebra homomorphisms. Then by a result of N. Muraki [Mur1, Mur2], under a normalization assumption, there are exactly 5 possibilities for a natural product: the tensor, Boolean, free and monotone/anti-monotone products.

A natural product is equivalent to a family $\sigma_{\mathcal{A}_{1}, \mathcal{A}_{2}}$ of linear mappings

$$
\sigma=\sigma_{\mathcal{A}_{1}, \mathcal{A}_{2}}: \mathcal{A}_{1} \sqcup \mathcal{A}_{2} \rightarrow \mathrm{S}\left(\mathcal{A}_{1} \oplus \mathcal{A}_{2}\right) \cong \mathrm{S}\left(\mathcal{A}_{1}\right) \otimes \mathrm{S}\left(\mathcal{A}_{2}\right) .
$$

via

$$
\varphi_{1} \bullet \varphi_{2}=\left(\mathrm{S}\left(\varphi_{1}\right) \otimes \mathrm{S}\left(\varphi_{2}\right)\right) \circ \sigma
$$

where $\mathrm{S}(V)$ is the unital symmetric tensor algebra over the vector space $V$. The axioms (1)-(3) can be translated to the family $\sigma_{\mathcal{A}_{1}, \mathcal{A}_{2}}$ :

$$
\begin{aligned}
\sigma_{\mathcal{A}_{1}, \mathcal{A}_{2}} \circ \iota_{1,2} & =\iota_{\mathcal{A}_{1} / \mathcal{A}_{2}} \\
\left(\mathrm{~S}\left(\sigma_{\mathcal{A}_{1}, \mathcal{A}_{2}}\right) \otimes \mathrm{id}\right) \circ \sigma_{\mathcal{A}_{1} \sqcup \mathcal{A}_{2}, \mathcal{A}_{3}} & =\left(\mathrm{id} \otimes \mathrm{S}\left(\sigma_{\mathcal{A}_{2}, \mathcal{A}_{3}}\right)\right) \circ \sigma_{\mathcal{A}_{1}, \mathcal{A}_{2} \sqcup \mathcal{A}_{3}} \\
\sigma_{\mathcal{B}_{1}, \mathcal{B}_{2}} \circ\left(j_{1} \sqcup j_{2}\right) & =\left(\mathrm{S}\left(j_{1}\right) \otimes \mathrm{S}\left(j_{2}\right)\right) \circ \sigma_{\mathcal{A}_{1}, \mathcal{A}_{2}}
\end{aligned}
$$

We have the functor $\mathrm{S}$ from the category of algebras to the category of unital commutative algebras, and a universal product is a family of maps

$$
\sigma_{\mathcal{A}_{1}, \mathcal{A}_{2}}: \mathcal{A}_{1} \sqcup \mathcal{A}_{2} \rightarrow \mathrm{S}\left(\mathcal{A}_{1}\right) \otimes \mathrm{S}\left(\mathcal{A}_{2}\right)
$$

from coproduct to coproduct satisfying (4)-(6); see [BGSch2].

\section{REFERENCES}

[BGSch1] Ben Ghorbal, A., Schürmann, M.: Non-commutative notions of stochastic independence. Math. Proc. Camb. Phil. Soc. 133, 531-561 (2002)

[BGSch2] Ben Ghorbal, A., Schürmann, M.: Quantum Lévy processes on dual groups. Math. Z. $251,147-165(2005)$

[Fra1] Franz, U.: Lévy processes on quantum groups and dual groups. In: Quantum Independent Increment Processes II, Lect. Notes Math., vol. 1866, Springer 2006

[Fra3] Franz, U.: Unification of boolean, monotone, anti-monotone and tensor independence and Lévy processes. Math. Z. 243, 779-816 (2003)

[Mur1] Muraki, N.: The five independences as quasi-universal products. IDAQP 5, 113-134 (2002)

[Mur2] Muraki, N.: The five independences as natural products. IDAQP 6, 337-371 (2003) 
[Sch] Schürmann, M.: Direct sums of tensor products and non-commutative independence. J. Funct. Anal. 133, 1-9 (1995)

[SchSkV] M. Schürmann, M. Skeide, S. Volkwardt: Transformations of Lévy processes. arXiv:0712.3504v2

[Sp2] Speicher, R.: On universal products. Fields Inst. Commun. 12, 257-266 (1997)

[SchVo] M. Schürman, S. Voß: Positivity of free convolution semigroups. Proceedings of the conference Quantum Probability Related Topics, Guanajuato, Mexico 2007

[Voi] D. Voiculescu: Dual algebraic structures on operator algebras related to free products. J. Operator Theory 17, 85-98 (1987)

[vW] W. von Waldenfels: Ito solution of the linear quantum stochastic differential equation describing light emission and absorption. In: Accardi, L., Frigerio, A., Gorini, V. (eds.) Quantum probability and applications to the theory of irreversible processes. Proceedings, Villa Mondragone 1982, Lect. Notes Math., vol. 1055, Springer 1984

\section{Noncommutative De Finetti Theorems and Noncommutative Independence}

Claus KÖSTLER

The classical de Finetti theorem characterizes an exchangeable infinite sequence of random variables to be identically distributed and conditionally independent over its tail algebra. It is a foundational result for the subject of distributional symmetries and invariance principles. Since we have still an insufficient understanding of noncommutative independence (including its conditional versions), a transfer of this result to noncommutative probability is of great interest. We introduce to related progress recently made in $[20,12,21,22]$.

Our starting point is a sequence of random variables $\mathcal{J}$, given by the embeddings

$$
\left(\iota_{n}\right)_{n \geq 0}:\left(\mathcal{A}_{0}, \varphi_{0}\right) \rightarrow(\mathcal{A}, \varphi) .
$$

Here $\left(\mathcal{A}_{0}, \varphi_{0}\right)$ and $(\mathcal{A}, \varphi)$ are two (noncommutative) probability spaces, each consisting of a von Neumann algebra and a faithful normal state. (We suppress some modular conditions needed if $\varphi$ is non-tracial.) It is immediate that such a sequence is identically distributed. Also the notions of the distributional symmetries 'exchangeability', 'spreadability' (a.k.a. 'contractiblity' or 'subsymmetry') and 'stationarity' transfer straightforward from probability to present noncommutative setting (see [20] for details). Now the main result of [20] is a noncommutative extended de Finetti theorem which shows that these symmetries yield noncommutative conditional independence, more general than those permitted by natural universality rules (see the talk of M. Schürmann). Actually the following notion of noncommutative conditional independence can be seen to emerge from these distributional symmetries.

Definition 1 (Noncommutative conditional independence [20]). The sequence J is said to be full $\mathcal{T}$-independent if

$$
E_{\mathcal{T}}(x y)=E_{\mathcal{T}}(x) E_{\mathcal{T}}(y)
$$

for $x \in \operatorname{vN}\left\{\mathcal{T}, \iota_{i}\left(\mathcal{A}_{0}\right) \mid i \in I\right\}$ and $y \in \operatorname{vN}\left\{\mathcal{T}, \iota_{j}\left(\mathcal{A}_{0}\right) \mid j \in J\right\}$ whenever $I$ and $J$ are disjoint subsets of $\mathbb{N}_{0}=\{0,1,2, \ldots\}$. 
Here $\operatorname{vN}\left\{\mathcal{T}, \iota_{i}\left(\mathcal{A}_{0}\right) \mid i \in I\right\}$ means the von Neumann algebra generated by $\mathcal{T}$ and $\left\{\iota_{i}\left(\mathcal{A}_{0}\right) \mid i \in I\right\}$. Moreover $E_{\mathcal{T}}$ denotes the $\varphi$-preserving conditional expectation from $\mathcal{A}$ onto a von Neumann subalgebra $\mathcal{T}$ of $\mathcal{A}$. In the context of de Finetti type results, $\mathcal{T}$ is given by the tail algebra of the considered sequence $\mathfrak{J}$ :

$$
\mathcal{T}=\bigcap_{n \geq 0} \operatorname{vN}\left\{\iota_{k}\left(\mathcal{A}_{0}\right) \mid k \geq n\right\} .
$$

We remark that $\mathcal{T}$ may be not commutative and, of course, $\mathcal{T}$ may be not contained in the von Neumann algebra generated by the ranges of finitely many random variables $\iota_{i}$.

Theorem 2 (Noncommutative extended de Finetti theorem [20]). Given the sequence $\mathfrak{J}$ consider the following conditions:

(E) $\mathcal{J}$ is exchangeable;

(S) J is spreadable;

(b) $\mathcal{J}$ is stationary and full $\mathcal{T}$-independent;

(a) $\mathcal{J}$ is identically distributed and full $\mathcal{T}$-independent.

Then it holds $(E) \Rightarrow(S) \Rightarrow(b) \Rightarrow(a)$, but each of the converse implications fails.

These four conditions are equivalent if the ranges of the random variables commute and, in this case, one recovers a dual version of E. Størmer's pioneering results on symmetric states on tensor products of $\mathrm{C}^{*}$-algebras [29]. If the underlying von Neumann algebra $\mathcal{A}$ is commutative, i.e. comes from a classical probability space, then Theorem 2 reduces to the usual extended de Finetti theorem, rewritten in an algebraic language.

One might object that a quantized result of de Finetti type should provide the equivalence of all four conditions (E) to (a), in particular since the result of RyllNadzewski [27] ensures the equivalence of (E) and (S) in the classical setting. But such common folklore from classical probability would be misleading in the quantum world. It is a well known phenomena in physics that one-dimensional quantum statistical models correspond to twodimensional classical ones [10]. So Theorem 2 should better be compared to results on classical random arrays, where exchangeability and spreadability are known to be inequivalent [18]. On the mathematical side and as explicated in the introduction of [20], each of the three inverse implications in Theorem 2 fails due to a deep structural reason (see also [20], Theorems 5.1 $\& 9.2]$ ). The implication (a) $\Rightarrow$ (b) fails because noncommutative independence in the sense of Definition 1 permits examples which are not captured by universality rules (compare the talk of $\mathrm{M}$. Schürmann). A much better understanding of these broken equivalences comes from the work [12] on braided random objects which I address next.

A new way towards a probabilistic interpretation of braid groups is taken in [12]. There we look at braided structures from the perspective of distributional symmetries and invariance principles. The guiding idea is that, as representations of the symmetric group $\mathbb{S}_{\infty}$ are connected to exchangeability, the representations of the braid group $\mathbb{B}_{\infty}$ should be connected to a new symmetry which we call braidability. 
The realization of this idea rests on two important pillars, the noncommutative extended de Finetti theorem, Theorem 2, and product representations of endomorphisms in operator theory [13].

Definition 3 (Braidability [12]). The random sequence $\mathcal{J}$ is braidable if there exists a representation of the braid group, $\rho: \mathbb{B}_{\infty} \rightarrow \operatorname{Aut}(\mathcal{A}, \varphi)$, such that

$$
\begin{aligned}
& \iota_{n}=\rho\left(\sigma_{n} \sigma_{n-1} \cdots \sigma_{1}\right) \iota_{0} \quad(n \in \mathbb{N}) \\
& \iota_{0}=\rho\left(\sigma_{n}\right) \iota_{0} \quad(n \geq 2)
\end{aligned}
$$

Here denotes $\sigma_{i}$ the Artin generators of $\mathbb{B}_{\infty}$ satisfying the relations $\sigma_{i} \sigma_{i+1} \sigma_{i}=$ $\sigma_{i+1} \sigma_{i} \sigma_{i+1}$ and $\sigma_{i} \sigma_{j}=\sigma_{j} \sigma_{i}$ if $|i-j|>1$. Moreover, $\operatorname{Aut}(\mathcal{A}, \varphi)$ denotes the $\varphi$-preserving ${ }^{*}$-automorphisms of $\mathcal{A}$. Note also that Definition 3 provides an extension of exchangeability: $\mathcal{J}$ is exchangeable if and only if $\mathcal{J}$ is braidable and $\rho\left(\sigma_{i}^{2}\right)=\mathrm{id}$ for $i \in \mathbb{N}$.

Our key result inserts braidability between the distributional symmetries (E) and $(\mathrm{S})$ :

Theorem 4 (Braided extended de Finetti theorem [12]). Consider the following additional property in Theorem 2:

(B) $\mathcal{J}$ is braidable.

Then one has $(\mathrm{E}) \Rightarrow(\mathrm{B}) \Rightarrow(\mathrm{S})$.

This refinement makes it clear that (E) and (S) are inequivalent in a 'quantized' framework, since otherwise representations of $\mathbb{B}_{\infty}$ would automatically be representations of $\mathbb{S}_{\infty}$. Most importantly, since exchangeability and spreadability are 'purely distributional' symmetries, Theorem 4 endows braid groups with a new intrinsic probabilistic interpretation. We emphasize this by a braided Hewitt-Savage Zero-One Law (see [12, Theorem 2.5]).

The results in [12] shed fresh light onto braid group representations [5, 9] as well as on Jones subfactor theory $[17,11]$. We illustrate this by means of the braid group von Neumann algebra $L\left(\mathbb{B}_{\infty}\right)$, a non-hyperfinite $I I_{1}$-factor non-isomorphic to all free group von Neumann algebras $L\left(\mathbb{F}_{n}\right)$, and study an irreducible subfactor inclusion of infinite Jones index (see [12, Theorem 5.2 and Corollary 5.3]). Our investigation reveals that the left regular representation of Artin generators provides a sequence which is neither braidable nor spreadable, but identically distributed and full $\mathbb{C}$-independent (see [12, Theorem 5.6]). This result prompts our search for another presentation which we call the square root of free generator presentation (see [12, Theorem 4.1]). We show that these new generators give rise to braidable sequences (see [12, Theorem 5.9]) and that their squares are a free system of Haar unitaries in the sense of Voiculescu. Additional considerations of random walks on groups [19] lead us to the speculation that combinatorial free probability [26] has a braided extension.

Some concrete examples from subfactor theory, among them Hecke algebras, show how this new approach allows to simplify some arguments. For this purpose we invoke noncommutative Bernoulli shifts (see [20, 12]). They correspond to sequences of 'quantized' random variables which are conditionally independent over 
their tail algebra ${ }^{1}$. Together with product representations of endomorphisms, these shifts provide us with powerful, flexible tools to analyze Galois type structures, in particular coming from braidable random sequences.

The long list of parallel results in classical probability and free probability $[32,31]$ suggests that there should also be a free version of de Finetti's theorem ${ }^{2}$. It is immediate from the noncommutative extended de Finetti theorem, Theorem 2 , that many different models of 'quantized' conditionally i.i.d. sequences enjoy exchangeability. So exchangeability as an invariance principle is unable to distinguish between them in the full generality of the present 'quantized' setting.

A new idea of how to strengthen exchangeability comes from the fact that exchangeability is not only a distributional symmetry (by its very definition), but also a dynamical symmetry: for all $n \in \mathbb{N}$, there exists a (state-preserving) action of the symmetric group $\mathbb{S}_{n}$. Now this action of $\mathbb{S}_{n}$ can equally well be expressed as a coaction of the commutative Hopf $\mathrm{C}^{*}$-algebras $C\left(\mathbb{S}_{n}\right)$ of continuous functions on $\mathbb{S}_{n}$. Doing so one can reformulate exchangeability as an invariance property of this coaction. This observation is a starting point of [22] and the crucial idea is now to replace 'permutations' by 'quantum permutations':

Wang introduces in [33] the quantum permutation group $A_{s}(n)$ as the universal quantum automorphism group acting on the $\mathrm{C}^{*}$-algebra $C(\{1, . ., n\})$ of continuous functions on $n$ points, answering a question of Connes. The $\mathrm{C}^{*}$-algebra $A_{s}(n)$ is a compact quantum group in the sense of Woronowicz [35] and contains $C\left(\mathbb{S}_{n}\right)$ as ordinary automorphisms on $C(\{1, \ldots, n\})$, i.e. as permutation matrices.

As exchangeability is an invariance property under the coaction of all $C\left(\mathbb{S}_{n}\right)$ 's, quantum exchangeability is introduced in [22] as an invariance property under the coaction of all $A_{s}(n)$ 's. This leads us to a new characterization of freeness with amalgamation:

Theorem 5 (Free de Finetti theorem [22]). The following are equivalent for an infinite sequence of random variables $\mathrm{J}$ :

(a) J is quantum exchangeable.

(b) $\mathcal{J}$ is identically distributed and free over its tail algebra.

To our knowledge this is the first result where a quantum group is considered as a quantum probabilistic invariant. Free independence has so far mainly been used as a tool in the study and classification of certain quantum groups [3, 2, 6]. On the other hand, actions of quantum groups on noncommutative spaces appear in subfactor theory (e.g. $[34,14,1,30]$ ), random walks on noncommutative spaces (e.g. [4, 7, 15, 16]) and in examples for noncommutative Bernoulli shifts [28]. Together with Theorem 2 and Theorem 4, the characterization result in Theorem 5

\footnotetext{
${ }^{1}$ Due to the broad concept of independence, our notion of a noncommutative Bernoulli shift is more general than shifts on tensor products or free products, in particular the one considered for the Connes-Størmer dynamical entropy [8, 25].

${ }^{2} \mathrm{~A}$ first attempt in this direction is undertaken by $\mathrm{F}$. Lehner, based on a cumulant approach via exchangeability systems [23, 24]. But the investigations in [21] reveal that weak freeness (as introduced in [24]) implies already freeness with amalgamation in a stationary setting.
} 
hints at a very deep connection between quantum symmetries and noncommutative conditional independence.

\section{REFERENCES}

[1] T. Banica. Representations of compact quantum groups and subfactors. J. Reine Angew. Math., 509:167-198, 1999.

[2] T. Banica, J. Bichon, and B. Collins. Quantum permutation groups: a survey. Banach Center Publ., 78:13-34, 2007.

[3] T. Banica and B. Collins. Integration over quantum permutation groups. J. Funct. Anal., 242:641-657, 2007.

[4] P. Biane. Quantum random walk on the dual of $\mathrm{su}(n)$. Probab. Theory Related Fields, 89(1):117-129, 1991.

[5] J. Birman. Braids, Links and Mapping Class Groups. Princeton University Press, 1975.

[6] T. Banica and R. Speicher. Liberation of orthogonal Lie groups. Preprint, 2008. (electronic) arXiv:0808.2628 [math.QA].

[7] B. Collins. Martin boundary theory of some quantum random walks. Ann. Inst. H. Poincaré Probab. Statist., 40(3):367-384, 2004.

[8] A. Connes and E. Størmer. Entropy for automorphisms of $I I_{1}$ von Neumann algebras. Acta Math., 134(3-4):289-306, 1975.

[9] P. Dehornoy. Braids and self distributivity, volume 192 of Progress in Mathematics. Birkhäuser Verlag, 2000.

[10] D.E. Evans and Y. Kawahigashi. Quantum symmetries on operator algebras. Oxford Mathematical Monographs. Oxford Science Publications. Oxford University Press, New York, 1998.

[11] F.M. Goodman, P. de la Harpe, and V.F.R. Jones. Coxeter Graphs and Towers of Algebras. Springer-Verlag, 1989.

[12] R. Gohm and C. Köstler. Noncommutative independence from the braid group $\mathbb{B}_{\infty}$. Commun. Math. Phys. To appear. Preprint (electronic) arXiv:0806.3691 [math.OA].

[13] R. Gohm. Noncommutative Stationary Processes, volume 1839 of Lecture Notes in Mathematics. Springer, 2004.

[14] T. Hayashi. Galois quantum groups of $\mathrm{II}_{1}$-subfactors. Tohoku Math. J. (2), 51(3), 1999.

[15] M. Izumi. Non-commutative Poisson boundaries. Contemp. Math., 347:69-81, 2004.

[16] W. Jaworski and M. Neufang. The Choquet-Deny equation in a Banach space. Canad. J. Math., 59(4):795-827, 2007.

[17] V.F.R. Jones. Index of subfactors. Invent. Math., 72:1-25, 1983.

[18] O. Kallenberg. Probabilistic Symmetries and Invariance Principles. Probability and Its Applications. Springer-Verlag, 2005.

[19] H. Kesten. Symmetric random walks on groups. Trans. Amer. Math. Soc., 92:336-354, 1959.

[20] C. Köstler. A noncommutative extended de Finetti theorem. J. Funct. Anal. To appear. Preprint (electronic) arXiv:0806.3621 [math.OA].

[21] C. Köstler. On Lehner's 'free' noncommutative de Finetti theorem. Proc. Amer. Math. Soc. To appear. Preprint (electronic) arXiv:0806.3632 [math.OA].

[22] C. Köstler and R. Speicher. A noncommutative de Finetti theorem: Invariance under quantum permutations is equivalent to freeness with amalgamation. Commun. Math. Phys. To appear. (electronic) arXiv:0807.0667 [math.OA].

[23] F. Lehner. Cumulants in noncommutative probability theory I. Noncommutative exchangeability systems. Math. Z., 248(1):67-100, 2004.

[24] F. Lehner. Cumulants in noncommutative probability theory IV. Noncrossing cumulants: De Finetti's theorem and $L^{p}$-inequalities. J. Funct. Anal., 239:214-246, 2006.

[25] S. Neshveyev and E. Størmer. Dynamical entropy in operator algebras. Ergebnisse der Mathematik und ihrer Grenzgebiete. 3. Folge. A Series of Modern Surveys in Mathematics. Springer-Verlag, Berlin, 2006. 
[26] A. Nica and R. Speicher. Lectures on the combinatorics of free probability, volume 335 of London Mathematical Society Lecture Note Series. Cambridge University Press, 2006.

[27] C. Ryll-Nardzewski. On stationary sequences of random variables and the de finetti's equivalence. Colloq. Math., 4:149-156, 1957.

[28] C. Rupp. Non-Commutative Bernoulli Shifts on Towers of von Neumann Algebras. PhD thesis, Univ. Tübingen, 1995. Dissertation.

[29] E. Størmer. Symmetric states of infinite tensor products of $\mathbf{C}^{*}$-algebras. J. Funct. Anal., 3:48-68, 1969.

[30] D. Shlyakhtenko and Y. Ueda. Irreducible subfactors of $L\left(\mathbb{F}_{\infty}\right)$ of index $\lambda>4$. J. Reine Angew. Math., 548:149-166, 2002.

[31] D.V. Voiculescu, K.J. Dykema, and A. Nica. Free Random Variables, volume 1 of CRM Monograph Series. American Math. Society, 1992.

[32] D. Voiculescu. Symmetries of some reduced free product C*-algebras. In S. Stratila H. Araki, C.C. Moore and D. Voiculescu, editors, Operator Algebras and their Connection with Topology and Ergodic Theory, volume 1132 of Lecture Notes in Mathematics, pages 556-588. Springer, 1985.

[33] S. Wang. Quantum symmetry groups of finite spaces. Commun. Math. Phys., 195:195-211, 1998.

[34] H. Wenzl. Quantum groups and subfactors of type B, C, and D. Comm. Math. Phys., 133(2):383-432, 1990.

[35] S.L. Woronowicz. Compact matrix pseudogroups. Commun. Math. Phys., 111:613-665, 1987.

\section{Research Talks — Brief Summary}

While the lectures served to give an overview over the state of the art about some well specified aspects relevant to the workshop, the talks gave occasion to present also recent and tentative news. The talks by Izumi and by Sinha deal with type III Arveson systems. The talks by Shalit, by Skalski, and by Accardi discuss versions different from one-parameter semigroups (the former two moreparameter semigroups, the latter a general nonstationary situation). The talks by Köstler, by Skeide, by von Waldenfes, and by Schürmann are in some sense related to independence. The talks by Tsirelson and by Bhat deal with specific problems of type II Arveson systems. The talk by Fagnola discusses the role of form generators.

\section{Generalized CCR Flows}

\section{MASAKI IZUMI}

(joint work with R. Srinivasan)

An $E_{0}$-semigroup is a weakly continuous semigroup of unital *-endomorphisms of $\mathbb{B}(H)$, where $H$ is a separable infinite dimensional Hilbert space. The notion of a product system was introduced by W. Arveson in his study of $E_{0}$-semigroups. He showed, on one hand, that the product system associated with an $E_{0}$-semigroup completely determines the cocycle conjugacy class of the $E_{0}$-semigroup, and on the other hand, that every product system arises from an $E_{0}$-semigroup (see [1]). 
Therefore classification of $E_{0}$-semigroups up to cocycle conjugacy is equivalent to that of product systems up to isomorphism.

Product systems, and hence $E_{0}$-semigroups, are classified into three categories, type I, type II, and type III, according to abundance of units. Type I $E_{0^{-}}$ semigroups are completely classified, and they are cocycle conjugate to so called CCR flows. By definition, type III $E_{0}$-semigroups have no units, and it is not so easy to construct them. Indeed, before Tsirelson's construction of uncountably many type III product systems [8], Powers' type III $E_{0}$-semigroup [7] was the only example. The main purpose of this talk is to report on the recent development of type III $E_{0}$-semigroups.

In [5], we showed that there exist uncountably many mutually non-isomorphic type III product systems that are not distinguished from type I product systems by Tsirelson's invariant. The invariant we employ is the von Neumann algebra $\mathcal{A}_{U}$ associated with an open subset $U$ of $[0,1]$, which is either a type I factor or a type III factor in our case.

Inspired by Tsirelson's construction of infinitely many mutually non-isomorphic type III product systems [8], Bhat and Srinivasan [2] introduced the notion of a sum system, which is a sort of "logarithm" of a product system giving rise to a product system via the Bosonic second quantization procedure. They obtained a dichotomy result about types, which says that every product system arising from a divisible sum system is either of type I or of type III.

One of the purposes of this talk is to show that every sum system is indeed divisible (see [4] for details). The proof goes through the notion of generalized CCR flows [5], which include the $E_{0}$-semigroups corresponding to Tsirelson's type III product systems [3]. In particular, every generalized CCR flow is either of type I or type III.

I also announce our recent result [6] showing that Powers' CAR construction also produces uncountably mutually non-cocycle conjugate type III $E_{0}$-semigroups.

\section{REFERENCES}

[1] W. Arveson, Non-commutative dynamics and E-semigroups, Springer Monograph in Math. (Springer 2003).

[2] B. V. R. Bhat and R. Srinivasan, On product systems arising from sum systems Infinite dimensional analysis and related topics, Vol. 8, Number 1, March 2005.

[3] M. Izumi, A perturbation problem for the shift semigroup. J. Funct. Anal. 251, (2007), $498-545$.

[4] M. Izumi, Every sum system is divisible, to appear in Trans. A.M.S. arXiv:0708.1591 [math.OA].

[5] M. Izumi and R. Srinivasan, Generalized CCR flows. Commun. Math. Phys. 281, (2008), $529-571$.

[6] M. Izumi and R. Srinivasan, in preparation.

[7] R. T. Powers, A nonspatial continuous semigroup of $*$-endomorphisms of $B(H)$, Publ. Res. Inst. Math. Sci. 23 (1987), 1053-1069.

[8] B. Tsirelson, Non-isomorphic product systems. Advances in Quantum Dynamics (South Hadley, MA, 2002), 273-328, Contemp. Math., 335, Amer. Math. Soc., Providence, RI, 2003. 


\section{Subproduct Systems and Dilation Theory of CP-Semigroups OrR MOShe Shalit}

(joint work with Baruch Solel)

The classical theory of isometric dilations deals with the following problem: given a semigroup of contractions $T=\left\{T_{s}\right\}_{s \in \mathcal{S}}$ acting on a Hilbert space $H$ (S $\mathcal{S}$ is some semigroup), find a semigroup $V=\left\{V_{s}\right\}_{s \in \mathcal{S}}$ of isometries acting on a Hilbert space $K \supseteq H$ such that for all $s \in \mathcal{S}$,

$$
T_{s}=\left.P_{H} V_{s}\right|_{H}
$$

This theory is highly developed [16] and well known. It is desirable to obtain a parallel theory for $*$-endomorphic dilations of semigroups of completely positive maps.

Let $\mathcal{S}$ be some sub-semigroup of $\mathbb{R}_{+}^{k}$, and let $\mathcal{M} \subseteq B(H)$ be a von Neumann algebra. A $C P$-semigroup (over $\mathcal{S}$ ) is a semigroup of $\mathrm{CP}$ maps, that is, a family $\Theta=\left\{\Theta_{s}\right\}_{s \in \mathcal{S}}$ of completely positive, contractive and normal maps on $\mathcal{M}$ such that

$$
\Theta_{s+t}(a)=\Theta_{s}\left(\Theta_{t}(a)\right), s, t \in \mathcal{S}, a \in \mathcal{M}
$$

and

$$
\Theta_{0}(a)=a, a \in \mathcal{M}
$$

When $\mathcal{S}$ has a topology we also require that the functions $s \mapsto\left\langle\Theta_{s}(a) h, g\right\rangle$ be continuous for all $a \in \mathcal{M}, h, g \in H$. A $C P_{0}$-semigroup is a semigroup of unital CP maps. An E-semigroup is a semigroup of $*$-endomorphisms. An $E_{0}$-semigroup is a semigroup of unital $*$-endomorphisms.

An E-dilation of $\Theta$ is a triple $(\alpha, K, \mathcal{R})$ consisting of a Hilbert space $K \supseteq H$ (with orthogonal projection $P_{H}: K \rightarrow H$ ), a von Neumann algebra $\mathcal{R} \subseteq B(K)$ that contains $\mathcal{M}$ as a corner $\mathcal{M}=P_{H} \mathcal{R} P_{H}$, and an E-semigroup $\alpha=\left\{\alpha_{s}\right\}_{s \in \mathcal{S}}$ on $\mathcal{R}$ such that for all $T \in \mathcal{R}, s \in \mathcal{S}$,

$$
\Theta_{s}\left(P_{H} T P_{H}\right)=P_{H} \alpha_{s}(T) P_{H} .
$$

The assertion that for every CP-semigroup there exists an E-dilation when $\mathcal{S}=\mathbb{N}$ or $\mathbb{R}_{+}$is called Bhat's Theorem, and appears in this form in [4] (under the additional restrictions that the semigroup is unital and that $\mathcal{M}=B(H)$ ). This results was reproved in $[9,6,8,3]$ (in greater generality).

In [5] and [15] appeared the result that for every CP-semigroup over the semigroup $\mathcal{S}=\mathbb{N}^{2}$ there exists an E-dilation.

The purpose of my lecture is to report on my (together with Baruch Solel) contributions to dilation theory of CP-semigroups, and to describe the general framework by which these results were obtained, and which I believe can underly a general dilation theory of CP-semigroups.

The reported contributions are these:

(1) For the case $\mathcal{S}=\mathbb{R}_{+}^{2}$ : under a technical assumption of strong commutativity, every CP-semigroup on $B(H)$ has an E-dilation, and every 
$\mathrm{CP}_{0}$-semigroup on an arbitrary von Neumann algebra has an $\mathrm{E}_{0}$-dilation $[11,12]$.

(2) For the case $\mathcal{S}=\mathbb{N}^{k}, k>2$ : in general there does not have to exist an E-dilation. We have an example of three commuting $\mathrm{CP}$ maps that have no (commuting) *-endomorphic dilation [13].

(3) For the case $\mathcal{S}=\mathbb{N}^{k}, k>2$ : (under the annoying technical assumption that the $\mathrm{CP}$ maps are of finite index and act on $B(H)$ ) if the semigroup is unit preserving, or if the generators are "small enough in norm", then there exist an E-dilation [13].

As a framework for obtaining the above results, we used subproduct systems and their representations (these notions have been formalized in [13], but they originate from Arveson's works [1] and [2]).

A subproduct system (over a von Neumann algebra $\mathcal{M}$ ) is a bundle of $\mathcal{M}$ correspondences $X=\{X(s)\}_{s \in \mathcal{S}}$, with $X(0)=\mathcal{M}$, such that there exist coisometric bimodule maps

$$
U_{s, t}: X(s) \otimes X(t) \rightarrow X(s+t)
$$

that compose associatively, i.e.,

$$
U_{s+t, r}\left(U_{s, t} \otimes I_{X(r)}\right)=U_{s, t+r}\left(I_{X(s)} \otimes U_{t, r}\right) .
$$

A representation of a subproduct system $X$ on a Hilbert space $G$ is a family of completely contractive maps $T=\left\{T_{s}\right\}_{s \in \mathcal{S}}, T_{s}: X(s) \rightarrow B(G)$ such that

(1) $T_{0}$ is a nondegenerate $*$-representation,

(2) For all $a, b \in \mathcal{M}, x \in X(s)$, we have $T_{s}(a x b)=T_{0}(a) T_{s}(x) T_{0}(b)$.

(3) For all $X \in X(s), y \in X(t)$, we have $T_{s+t}\left(U_{s, t}(x \otimes y)\right)=T_{s}(x) T_{t}(y)$.

We have shown in [13] that there is an essentially 1-1 correspondence between CP-semigroups and pairs $(X, T)$ where $X$ is a subproduct system and $T$ is a representation of $T$. This correspondence respects the notions of dilations, so the problem of dilating CP-semigroups to E-semigroups is equivalent to the problem of constructing "isometric dilations" to subproduct system representations. The latter problem may be handled using methods from classical isometric dilation theory, thus providing a foundation for a theory of dilation of CP-semigroups, which runs parallel to the classical theory of isometric dilations.

\section{REFERENCES}

[1] Wm. B. Arveson, Continuous analogues of Fock space, Mem. Amer. Math. Soc., No. 409, American Mathematical Society, 1989.

[2] Wm. B. Arveson, The index of a quantum dynamical semigroup, J. Funct. Anal., Vol. 146, No. 2 (1997), 557588.

[3] Wm.B. Arveson, Non commutative dynamics and E-semigroups, Springer Monographs in Math., Springer-Verlag, 2003.

[4] B. V. R. Bhat, An index theory for quantum dynamical semigroups, Trans. Amer. Math. Soc. Vol. 348, No. 2 (1996), 561-583.

[5] B. V. R. Bhat A generalized intertwining lifting theorem, in: Operator Algebras and Applications, II, Waterloo, ON, 1994-1995, in: Fields Inst. Commun., vol. 20, Amer. Math. Soc., Providence, RI, 1998, pp. 1-10. 
[6] B. V. R. Bhat and M. Skeide, Tensor product systems of Hilbert modules and dilations of completely positive semigroups, Infinite Dimensional Analysis, Quantum Probability and Related Topics, Vol. 3, (2000), 519-575.

[7] D. Markiewicz, On the product system of a completely positive semigroup, J. Funct. Anal.. Vol. 200, No. 1 (2003), 237-280.

[8] P. Muhly and B. Solel, Quantum Markov Processes (Correspondences and Dilations), Internat. J. Math. Vol. 13, No. 8 (2002), 863-906.

[9] D. SeLegue, Minimal Dilations of CP maps and $C^{*}$-Extension of the Szegö Limit Theorem, Ph.D Dissertation, University of California, Berkeley, 1997.

[10] O. M. Shalit, Representing a product system representation as a contractive semigroup and applications to regular isometric dilations, to appear in Canad. Math. Bull.

[11] O. M. Shalit, Every commuting pair of $C P$-semigroups on $M_{n}(\mathbb{C})$ has an E-dilation (and so do many others), preprint, arXiv:0711.2885v2 [math.OA], 2007.

[12] O. M. Shalit, E- Eilation of strongly commuting $C P_{0}$-semigroups, J. Funct. Anal., Vol. 255, No. 1 (2008), 46-89.

[13] O. M. Shalit and B. Solel, Subproduct systems, preprint, arXiv:0901.1422v1 [math.OA].

[14] M. Skeide, Product Systems; a Survey with Commutants in View, to appear in the proceedings of the 2006 Quantum Probability Conference in Nottingham.

[15] B. Solel, Representations of product systems over semigroups and dilations of commuting CP maps, J. Funct. Anal., Vol. 235, No. 2 (2006) 593-618.

[16] B. Sz.-Nagy and C. Foiaş, Harmonic Analysis of Operators in Hilbert Space, North-Holland, Amsterdam, 1970.

\section{Noncommutative Factorizations Claus KÖStler}

Homogeneous measure factorizations (or noises) canonically induce Arveson systems of Hilbert spaces (see $[9,8,7,1]$ ). If the measure factorization comes from Lévy-Kchinchin processes, the obtained Arveson product system are known to be of type I. Starting from non-classical noises as models for homogeneous measure factorizations, B. Tsirelson has established a rich source of Arveson systems of type II.

This talk reports about ongoing research on a noncommutative analogue of measure factorizations and their connection to Arveson systems [6]. The formulation of such an analogue is straightforward, based on noncommutative independence as it appears in $[5,2]$ or in [4].

Definition 1. Let $\mathcal{A}$ be a von Neumann algebra (with separable predual) equipped with the faithful normal state $\varphi$. A noncommutative factorization of the probability space $(\mathcal{A}, \varphi)$ over a (complete) Boolean algebra $\mathbb{B}$ is given by a family $\left(\mathcal{A}_{B}\right)_{B \in \mathbb{B}}$ of $\varphi$-conditioned von Neumann subalgebras $\mathcal{A}_{B}$ of $\mathcal{A}$ satisfying the following conditions:

(i) $\mathcal{A}_{B_{1} \wedge B_{2}}=\mathcal{A}_{B_{1}} \wedge \mathcal{A}_{B_{2}}$;

(ii) $\mathcal{A}_{B_{1} \vee B_{2}}=\mathcal{A}_{B_{1}} \vee \mathcal{A}_{B_{2}}$

(iii) $\mathcal{A}_{B_{1}}$ and $\mathcal{A}_{B_{2}}$ are $\mathbb{C}$-independent if $B_{1} \wedge B_{2}=0_{\mathbb{B}}$;

(iv) if $B_{n} \nearrow 1_{\mathbb{B}}$ then $\bigvee_{n} \mathcal{A}_{B_{n}}=\mathcal{A}$.

Further a continuity (or measureablity) condition is required if $\mathbb{B}$ is non-atomic. 
We are especially interested in the following choices of the Boolean algebra $\mathbb{B}$ : Lebesgue measureable sets in $\mathbb{R}$ or all subsets of $\mathbb{Z}$ (or their restrictions to $\mathbb{R}_{0}$ resp. $\left.\mathbb{N}_{0}\right)$. The noncommutative factorization is said to be homogeneous if there exists a group of $\varphi$-preserving automorphisms $\left(\alpha_{t}\right)_{t}$ of $\mathcal{A}$ such that $\alpha_{t}\left(\mathcal{A}_{B}\right)=\mathcal{A}_{B+t}$ for all $t \in \mathbb{R}$ (or $\mathbb{Z}$ ) and $B \in \mathbb{B}$. Note that the setting in Definition 1 is such that the usual notion of a (homogeneous) measure factorization can be recovered as soon as the von Neumann algebra $\mathcal{A}$ is commutative. In this case $\mathbb{C}$-independence reduces to stochastic independence in probability theory.

The underlying notion of noncommutative independence is very general and presently almost nothing is known about noncommutative factorizations in the sense of Definition 1. First investigations give strong evidence that there exists a rich theory of noncommutative factorizations waiting to be explored further.

Candidates for homogeneous noncommutative factorizations (over $\mathbb{R}$ ) are $\mathbb{C}$ expected white noises in the sense of [4, Definition 6.5.2]. Examples for the latter are, aside of classical Poisson or Gaussian white noise, CCR white noises, CAR white noises and $q$-Gaussian white noises (see Examples 6.6.3 to 6.6.5 in [4]). In these examples the von Neumann algebra $\mathcal{A}$ is generated by unitary (adapted) cocycles with respect to the time shift; and each of these unitary cocycles provides a normalized unit in the GNS Hilbert space of these noises.

Conjecture 2. Every $\mathbb{C}$-expected white noise (in the sense of [4]) gives rise to a noncommutative factorization.

These quantum white noises may be regarded as noncommutative analogues of Fock factorizations.

Question 3. Suppose $\mathcal{A}$ is a type $I_{1}$ or type III factor. Does there exist a homogeneous noncommutative factorization of $(\mathcal{A}, \varphi)$ (over $\mathbb{R}$ ) which leads to an Arveson sytem of type II?

Due to the underlying notion of independence, already the study of noncommutative factorizations over $\mathbb{Z}$ or $\mathbb{N}_{0}$ is highly non-trivial.

Very recently we have found the following example in the context of exchangeability. Let $\mathbb{S}_{\infty}$ be the group of all finite permutations on $\mathbb{N}_{0}$. We consider as probability space the group von Neumann algebra $L\left(\mathbb{S}_{\infty}\right)$ equipped with the normalized trace $\operatorname{tr}_{\infty}$. Let $\mathcal{A}_{I}=\operatorname{vN}\left\{L_{(0, k)} \mid k \in I \subset \mathbb{N}\right\}$, where $L_{(0, k)}$ is the left shift on the Hilbert space $\ell^{2}\left(\mathbb{S}_{\infty}\right)$ associated to the transposition $(0, k)$ on $\mathbb{N}_{0}$.

Theorem 4. The family $\left(\mathcal{A}_{I}\right)_{I \subset \mathbb{N}_{0}}$ is a noncommutative factorization of $\left(L\left(\mathbb{S}_{\infty}\right), \operatorname{tr}_{\infty}\right)$.

The proof of this result is based on exchangeability in a noncommutative setting [3]. We expect more generally:

Conjecture 5. Every minimal exchangeable random sequence with trivial tail algebra (in the sense of [5]) gives rise to a noncommutative factorization over $\mathbb{N}_{0}$.

\section{REFERENCES}

[1] W. Arveson. Noncommutative Dynamics and E-Semigroups. Springer Monographs in Mathematics. Springer-Verlag, 2003. 
[2] R. Gohm and C. Köstler. Noncommutative independence from the braid group $\mathbb{B}_{\infty}$ Commun. Math. Phys. To appear. Preprint (electronic) arXiv:0806.3691 [math.OA].

[3] R. Gohm and C. Köstler. Noncommutative independence from the symmetric group $\mathbb{S}_{\infty}$. Preprint in preparation.

[4] J. Hellmich, C. Köstler, and Kümmerer. Noncommutative continuous Bernoulli shifts. (electronic) arXiv: math.OA/0411565, 2004.

[5] C. Köstler. A noncommutative extended de Finetti theorem. J. Funct. Anal. To appear. Preprint (electronic) arXiv:0806.3621 [math.OA].

[6] C. Köstler. On the relationship between continuous Bernoulli systems, Tsirelson's continuous products of probability spaces and Arveson's product systems. In preparation.

[7] V. Liebscher. Random sets and invariants for (type II) continuous tensor product systems of Hilbert spaces. Preprint arXiv:math.PR/0306365, 2003.

[8] B. Tsirelson. Nonclassical stochastic flows and continuous products. Probab. Surv., 1:173-298, 2004. (electronic) arXiv: math.PR/0402431 v2.

[9] B. Tsirelson and A.V. Vershik. Examples of nonlinear continuous tensor products of measure spaces and non-Fock factorizations. Rev. Math. Phys., 10:81-145, 1998.

\section{Nonequivalent Units}

BORIS TSIRELSON

Theorem 1. There exists an Arveson system of type $I I_{1}$ such that the action of the group of automorphisms on the set of normalized units is not transitive.

In order to prove the theorem it is sufficient to construct a noise, extending the white noise and drift sensitive in the following sense: for every $\lambda \in \mathbb{R} \backslash\{0\}$ the extension obtained by the drift $\lambda$ is non-isomorphic to the original extension on the level of Arveson systems (that is, the corresponding extensions of the type $I_{1}$ Arveson system are non-isomorphic).

Here is why it is sufficient. In the group of all motions of the complex plane we consider the subgroup $G$ of motions that correspond to automorphisms of the type $I_{1}$ Arveson system $\left(H_{t}^{\text {white }}\right)_{t}$ extendable to the type $I I_{1}$ Arveson system $\left(H_{t}\right)_{t}$. Real shifts $z \mapsto z+\lambda$ (for $\lambda \in \mathbb{R} \backslash\{0\}$ ) do not belong to $G$ by the drift sensitivity. Imaginary shifts $z \mapsto z+\mathrm{i} \lambda$ (for $\lambda \in \mathbb{R}$ ) belong to $G$, since the operators $\Theta_{t}^{\text {shift }(\mathrm{i} \lambda \text { ) }}$ of multiplication by $\exp \left(\mathrm{i} \lambda B_{t}\right)$ act naturally on $H_{t}$. It follows that $G$ contains no rotations (except for the rotation by $\pi$ ) and therefore is not transitive.

The drift sensitive extension is achieved by attaching a random function $S$ : $(\tau, \infty) \rightarrow\{-1,+1\}$ to each (random) point $\tau$ of local minimum of the Brownian motion. The function $S$ is constant on $\left[\tau+2 \cdot 3^{-n-1}, \tau+2 \cdot 3^{-n}\right)$ for each $n$ and such that

$$
\frac{S\left(\tau+2 \cdot 3^{-n}\right)}{S\left(\tau+2 \cdot 3^{-n}-\right)}=f_{n}\left(B\left(\tau+2 \cdot 3^{-n}\right)-B(\tau)\right)
$$

for all $n$. Here $f_{n}: \mathbb{R} \rightarrow\{-1,+1\}$ are nonrandom functions chosen appropriately.

Definitions and statements given below are not really used in the proof of the theorem, but probably help to understand the idea. The phenomenon of a nonextendable isomorphism (as well as nonisomorphic extensions) is demonstrated 
here by a toy model, - a kind of product system of Hilbert spaces, simpler than Arveson system.

Definition 1. A toy product system of Hilbert spaces is a triple $\left(H_{1}, H_{\infty}, U\right)$, where $H_{1}, H_{\infty}$ are Hilbert spaces (over $\mathbb{C}$, separable), and $U: H_{1} \otimes H_{\infty} \rightarrow H_{\infty}$ is a unitary operator.

We treat it as a kind of product system, since

$$
H_{\infty} \sim H_{1} \otimes H_{\infty} \sim H_{1} \otimes H_{1} \otimes H_{\infty} \sim \ldots
$$

where ' $\sim$ ' means: may be identified naturally (using $U$ ).

An evident example: $H_{\infty}=\left(H_{1}, \psi_{1}\right)^{\otimes \infty}$ is the infinite tensor product of (an infinite sequence of) copies of $H_{1}$ relatively to (the copies of) a given vector $\psi_{1} \in$ $H_{1},\left\|\psi_{1}\right\|=1$.

A more interesting example: $H_{\infty}=\left(H_{1}, \psi_{1}\right)^{\otimes \infty} \oplus\left(H_{1}, \psi_{2}\right)^{\otimes \infty}$ is the direct sum of two such infinite tensor products, one relative to $\psi_{1}$, the other relative to another vector $\psi_{2} \in H_{1},\left\|\psi_{2}\right\|=1, \psi_{2} \neq \psi_{1}$.

Definition 2. Let $\left(H_{1}, H_{\infty}, U\right)$ and $\left(H_{1}^{\prime}, H_{\infty}^{\prime}, U^{\prime}\right)$ be toy product systems of Hilbert spaces. An isomorphism between them is a pair $\Theta=\left(\Theta_{1}, \Theta_{\infty}\right)$ of unitary operators $\Theta_{1}: H_{1} \rightarrow H_{1}^{\prime}, \Theta_{\infty}: H_{\infty} \rightarrow H_{\infty}^{\prime}$ such that the diagram

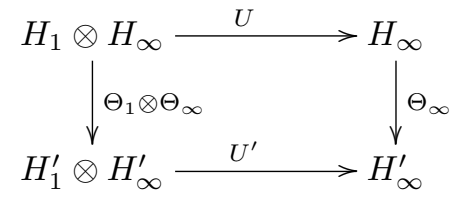

is commutative.

Thus,

$$
\Theta_{\infty} \sim \Theta_{1} \otimes \Theta_{\infty} \sim \Theta_{1} \otimes \Theta_{1} \otimes \Theta_{\infty} \sim \ldots
$$

A unitary operator $\Theta_{1}: H_{1} \rightarrow H_{1}$ leads to an automorphism of $\left(H_{1}, \psi_{1}\right)^{\otimes \infty}$ (that is, of the corresponding toy product system) if and only if $\Theta_{1} \psi_{1}=\psi_{1}$. Similarly, $\Theta_{1}$ leads to an automorphism of $\left(H_{1}, \psi_{1}\right)^{\otimes \infty} \oplus\left(H_{1}, \psi_{2}\right)^{\otimes \infty}$ if and only if either $\Theta_{1} \psi_{1}=\psi_{1}$ and $\Theta_{1} \psi_{2}=\psi_{2}$, or $\Theta_{1} \psi_{1}=\psi_{2}$ and $\Theta_{1} \psi_{2}=\psi_{1}$.

Taking $\Theta_{1}$ such that $\Theta_{1} \psi_{1}=\psi_{1}$ but $\Theta_{1} \psi_{2} \neq \psi_{2}$ we get an automorphism of $\left(H_{1}, \psi_{1}\right)^{\otimes \infty}$ that cannot be extended to an automorphism of $\left(H_{1}, \psi_{1}\right)^{\otimes \infty} \oplus$ $\left(H_{1}, \psi_{2}\right)^{\otimes \infty}$.

Definition 3. A toy product system of probability spaces is a triple $\left(\Omega_{1}, \Omega_{\infty}, \alpha\right)$, where $\Omega_{1}, \Omega_{\infty}$ are probability spaces (standard), and $\alpha: \Omega_{1} \times \Omega_{\infty} \rightarrow \Omega_{\infty}$ is an isomorphism mod 0 (that is, an invertible measure preserving map).

Every toy product system of probability spaces $\left(\Omega_{1}, \Omega_{\infty}, \alpha\right)$ leads to a toy product system of Hilbert spaces $\left(H_{1}, H_{\infty}, U\right)$ as follows:

$$
\begin{gathered}
H_{1}=L_{2}\left(\Omega_{1}\right) ; \quad H_{\infty}=L_{2}\left(\Omega_{\infty}\right) ; \\
(U \psi)(\cdot)=\psi\left(\alpha^{-1}(\cdot)\right) .
\end{gathered}
$$


Here we use the canonical identification

$$
L_{2}\left(\Omega_{1}\right) \otimes L_{2}\left(\Omega_{\infty}\right)=L_{2}\left(\Omega_{1} \times \Omega_{\infty}\right)
$$

and treat a vector $\psi \in H_{1} \otimes H_{\infty}$ as an element of $L_{2}\left(\Omega_{1} \times \Omega_{\infty}\right)$.

An evident example: $\Omega_{\infty}=\Omega_{1}^{\infty}$ is the product of an infinite sequence of copies of $\Omega_{1}$. It leads to $H_{\infty}=\left(H_{1}, \mathbb{1}\right)^{\otimes \infty}$ where $H_{1}=L_{2}\left(\Omega_{1}\right)$ and $\mathbb{1} \in L_{2}\left(\Omega_{1}\right)$ is the constant function, $\mathbb{1}(\cdot)=1$.

Here is a more interesting example. Let $X_{1}: \Omega_{1} \rightarrow\{-1,+1\}$ be a random variable (not a constant). We define $\Omega_{\infty}$ as the set of all double sequences $\left(\begin{array}{c}\omega_{1}, \omega_{2}, \ldots \\ s_{1}, s_{2}, \ldots\end{array}\right)$ such that $\omega_{k} \in \Omega_{1}, s_{k} \in\{-1,+1\}$ and $s_{k}=s_{k+1} X_{1}\left(\omega_{k}\right)$ for all $k$. Sequences $\left(\omega_{1}, \omega_{2}, \ldots\right) \in \Omega_{1}^{\infty}$ are endowed with the product measure. The conditional distribution of the sequence $\left(s_{1}, s_{2}, \ldots\right)$, given $\left(\omega_{1}, \omega_{2}, \ldots\right)$, must be concentrated on the two sequences obeying the relation $s_{k}=s_{k+1} X_{1}\left(\omega_{k}\right)$. We give to these two sequences equal conditional probabilities, 0.5 to each. Thus, $\Omega_{\infty}$ is endowed with a probability measure. The map $\alpha: \Omega_{1} \times \Omega_{\infty} \rightarrow \Omega_{\infty}$ is defined by

$$
\alpha\left(\omega_{1},\left(\begin{array}{ccc}
\omega_{2}, & \omega_{3}, & \ldots \\
s_{2}, & s_{3}, & \ldots
\end{array}\right)\right)=\left(\begin{array}{cccc}
\omega_{1}, & \omega_{2}, & \omega_{3}, & \ldots \\
s_{2} X_{1}\left(\omega_{1}\right), & s_{2}, & s_{3}, & \ldots
\end{array}\right) .
$$

Clearly, $\alpha$ is measure preserving.

This system $\left(\Omega_{1}, \Omega_{\infty}, \alpha\right)$ leads to a system $\left(H_{1}, H_{\infty}, U\right)$ of the form $\left(H_{1}, \psi_{1}\right)^{\otimes \infty} \oplus$ $\left(H_{1}, \psi_{2}\right)^{\otimes \infty}$ (up to isomorphism), as explained below. We have

$$
\begin{gathered}
H_{1}=L_{2}\left(\Omega_{1}\right), \quad H_{\infty}=L_{2}\left(\Omega_{\infty}\right), \\
(U \psi)\left(\begin{array}{cccc}
\omega_{1}, & \omega_{2}, & \omega_{3}, & \ldots \\
s_{1}, & s_{2}, & s_{3}, & \ldots
\end{array}\right)=\psi\left(\omega_{1},\left(\begin{array}{ccc}
\omega_{2}, & \omega_{3}, & \ldots \\
s_{2}, & s_{3}, & \ldots
\end{array}\right)\right) .
\end{gathered}
$$

The function $S: \Omega_{\infty} \rightarrow\{-1,+1\}$ defined by

$$
S\left(\begin{array}{cccc}
\omega_{1}, & \omega_{2}, & \omega_{3}, & \ldots \\
s_{1}, & s_{2}, & s_{3}, & \ldots
\end{array}\right)=s_{1}
$$

may be identified with $X_{1}^{\otimes \infty}$, since

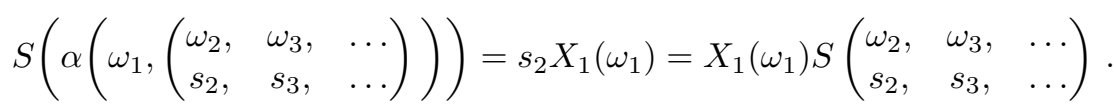

See also [2].

\section{REFERENCES}

[1] W. Arveson, Noncommutative dynamics and E-semigroups, Springer, New York, 2003.

[2] B. Tsirelson, On automorphisms of type II Arveson systems (probabilistic approach), New York Journal of Mathematics 14 (2008), 539-576. 


\section{Inclusion Systems and Amalgamated Products of Product Systems}

\section{B.V.R. BHAT}

Inclusion systems (or subproduct systems) are families of Hilbert spaces $\left\{E_{t}: t \geq\right.$ $0\}$ with an associative family of isometries $\left\{\beta_{s+t}: s, t \geq 0\right\}$ :

$$
\beta_{s, t}: E_{s+t} \rightarrow E_{s} \otimes E_{t} .
$$

Every inclusion system gives rise to a product system through an inductive limit procedure. We show that structures such as units and morphisms of these product systems can be determined at the level of inclusion systems. We use this idea to study Powers problem and Skeide product of spatial product systems. We determine the product system of 'Powers' sum through arbitrary corners' of two $\mathrm{CP}$ semigroups. This is a joint work with Mithun Mukherjee.

\section{REFERENCES}

[1] Bhat B.V. Rajarama and Mukherjee M., Inclusion systems and amalgamated products of product systems, preprint under preparation.

[2] Bhat B.V. Rajarama, Liebscher V., and Skeide M., A problem of Powers and the product of spatial product systems, In Proceedings of the 28th Conference on Quantum Probability and Related Topics,QP-PQ Vol. 23 (World Scientific). arXiv: 0801.0042v1, 2007.

[3] Bhat B.V. Rajarama, Liebscher V., and Skeide M., Two notions of product for continuous product systems of Hilbert spaces, preprint under preparation.

[4] Powers, R.T., Addition of spatial E0semigroups, Operator algebras, quantization, and noncommutative geometry, Contemporary Mathematics, no. 365, American Mathematical Society, 2004, pp. 281-298.

[5] Skeide, M., The Powers sum of spatial CPD-semigroups and CP-semigroups. arXiv: 0812.0077v1, 2009

\section{Quantum Markov Semigroups and Flows Arising from Form Generators on $\mathcal{B}(\mathbf{h})$}

FRANCO FAGNOLA

A form generator $£$ on $\mathcal{B}(\mathrm{h})$ is given by a family $£(x)$ of quadratic forms

$$
£(x)[v, u]=\langle G v, x u\rangle+\sum_{\ell \geq 1}\left\langle L_{\ell} v, x L_{\ell} u\right\rangle+\langle v, x G u\rangle
$$

where $x \in \mathcal{B}(\mathrm{h}), G$ is the generator of a strongly continuous semigroup $\left(P_{t}\right)_{t \geq 0}$ on h, $v, u \in \operatorname{Dom}(G)$, the $L_{\ell}$ are defined on $\operatorname{Dom}(G)$ and the algebraic condition for identity preservation $£(\mathbb{1})[v, u]=0$ holds.

This is an extension of the well-known Gorini-Kossakowski-Sudarshan-Lindblad representation of generators norm-continuous completely positive Markov semigroups on $\mathcal{B}(\mathrm{h})$ also called quantum dynamical semigroups.

A semigroup $\left(\mathcal{T}_{t}\right)_{t \geq 0}$ associated with the above quadratic should satisfy

$$
\left\langle v, \mathcal{T}_{t}(x) u\right\rangle=\langle v, x u\rangle+\int_{0}^{t} £\left(\mathcal{T}_{s}(x)\right)[v, u] d s
$$


for all $u, v \in \operatorname{Dom}(G)$ and $x \in \mathcal{B}(\mathrm{h})$. Computing the derivative of the function $s \rightarrow$ $\left\langle P_{t-s} v, \mathcal{T}_{s}(s) P_{t-s} u\right\rangle$ it is easy to see that a strongly continuous family $\left(\mathcal{T}_{t}(x)\right)_{t \geq 0}$ in $\mathcal{B}(\mathrm{h})$ solves (1) if and only if it satisfies

$$
\left\langle v, \mathcal{T}_{t}(x) u\right\rangle=\left\langle P_{t} v, x P_{t} u\right\rangle+\sum_{\ell=1}^{\infty} \int_{0}^{t}\left\langle L_{\ell} P_{t-s} v, \mathcal{I}_{s}(x) L_{\ell} P_{t-s} u\right\rangle d s .
$$

This leads naturally to the iteration scheme $\mathcal{T}_{t}^{(0)}(x)=P_{t}^{*} x P_{t}$ and

$$
\left\langle v, \mathcal{T}_{t}^{(n+1)}(x) u\right\rangle=\left\langle v, P_{t}^{*} x P_{t} u\right\rangle+\sum_{\ell=1}^{\infty} \int_{0}^{t}\left\langle L_{\ell} P_{t-s} v, \mathcal{T}_{s}^{(n)}(x) L_{\ell} P_{t-s} u\right\rangle d s
$$

It can be shown that, for $x$ positive, $\mathcal{T}_{t}^{(n+1)}(x) \uparrow \mathcal{T}_{t}(x)$ and $\left(\mathcal{T}_{t}\right)_{t \geq 0}$ is a weakly*continuous semigroup on $\mathcal{B}(\mathrm{h})$ (see e.g. [5] Sect.3). It is called the minimal semigroup because, if $\left(\mathcal{T}_{t}^{\prime}\right)_{t \geq 0}$ is another semigroup solving (1), then $\mathcal{T}_{t}(x) \leq \mathcal{T}_{t}^{\prime}(x)$ for all positive $x$. As a consequence, a priori we know only that $\mathcal{T}_{t}(\mathbb{1}) \leq \mathbb{1}$. Indeed, the semigroup semigroup solving (1) is unique if and only if $\mathcal{T}_{t}(\mathbb{1})=\mathbb{1}$.

This construction, and its pre-dual version of E.B. Davies in [4], generalises the minimal solution of Kolmogorov equations for classical time-continuous Markov chains (see e.g. [3]).

When the minimal semigroup $\mathcal{T}$ is identity preserving and the unbounded operators $G, L_{\ell}$ are well behaved (e.g. the intersection of their domains and the domains of their adjoints is an essential domain for $G$ and $G^{*}$, one can compose any pair of them on a certain dense domain and similar technical conditions) a dilation of $\mathcal{T}$ to a inner quantum flow of the form $j_{t}(X)=U_{t}^{*} X U_{t}$ ( $X$ operator on $\mathrm{h} \otimes \mathcal{F}$ ) can be constructed by means of Hudson-Parthasarathy quantum stochastic calculus. The operators $U_{t}$ are unitary cocycles with respect to the natural right shift on the Fock space $\mathcal{F}$ over $L^{2}\left(\mathbb{R}_{+} ; \mathrm{k}\right)$ and are the unique solution of a quantum stochastic differential equation driven by creation, annihilation and number processes whose coefficients are partially determined by the operators $G, L_{\ell}$.

In this talk we present concrete examples of form generators with "good" or singular operators $G, L_{\ell}$. Singular examples appear in the study of extensions of simple classical Markov semigroup on an abelian algebra to quantum Markov semigroups on a $\mathcal{B}(\mathrm{h})$ that contains it as a subalgebra (see e.g. [2] for the semigroup of a Brownian motion on a closed set with some boundary condition). We also illustrate the analytical obstructions that one can find in the construction of the unitary cocycle $\left(U_{t}\right)_{t \geq 0}$ and distinguish good cases from singular ones.

By their own construction minimal semigroups lead to a product system with units. One expects that, when the operators $G, L_{\ell}$ are well behaved, this product system is type I as it happens when $G$ and the $L_{\ell}$ are bounded and the $L_{\ell}$ are chosen to be linearly independent as proved by Bhat [1]. It would be interesting to know whether there are type II product system arising in the dilation of minimal semigroups. 


\section{REFERENCES}

[1] B.V.R. Bhat, Cocycles of CCR Flows, Memoirs of the American Mathematical Society 146 (2001).

[2] B.V.R. Bhat, F. Fagnola, K.B. Sinha, On quantum extensions of semigroups of brownian motions on an half-line, Russian J. Math. Phys. 4 (1996), 13-28.

[3] K.L. Chung, Markov Chains with Stationary Transition Probability Springer-Verlag, 1960.

[4] E.B. Davies, Quantum dynamical semigroups and the neutron diffusion equation, Rep. Math. Phys. 11 (1977), 169-188.

[5] F. Fagnola, Quantum Markov Semigroups and Quantum Markov Flows, Proyecciones 18, (1999), 1-144.

\section{Free Product Systems \\ Michael SKeIDE}

For terminology, notations, and references we refer to the introduction, which starts on Page 5.

Let $\mathcal{F}(F):=\bigoplus_{n=0}^{\infty} F^{\odot n}$ denote the full Fock module over the Hilbert $\mathcal{B}-\mathcal{B}$-bimodule $F$. The full Fock module to the direct sum $F_{1} \oplus F_{2}$ factors as

$$
\mathcal{F}\left(F_{1} \oplus F_{2}\right)=\mathcal{F}\left(F_{1}\right) \odot\left(\omega \mathcal{B} \oplus\left(F_{2} \odot \mathcal{F}\left(F_{1} \oplus F_{2}\right)\right)\right) .
$$

Let us define $E_{S}:=L^{2}(S, F)$ for any (measurable) subset $S$ of $\mathbb{R}_{+}$, and $E:=$ $E_{\mathbb{R}_{+}}$. For every $S$ and $T \supset S+t$ define the second quantized right shift isometry $s_{t}: \mathcal{F}\left(E_{S}\right) \rightarrow \mathcal{F}\left(E_{T}\right)$. Then the factorization

$$
\begin{aligned}
\mathcal{F}(E) & =\mathcal{F}\left(E_{[t, \infty)}\right) \odot\left(\omega \mathcal{B} \oplus\left(E_{[0, t)} \odot \mathcal{F}(E)\right)\right) \\
& \cong s_{t} \mathcal{F}\left(E_{[t, \infty)}\right) \odot\left(\omega \mathcal{B} \oplus\left(E_{[0, t)} \odot \mathcal{F}(E)\right)\right) \\
& =\mathcal{F}(E) \odot\left(\omega \mathcal{B} \oplus\left(E_{[0, t)} \odot \mathcal{F}(E)\right)\right)
\end{aligned}
$$

induces an $E_{0}$-semigroup $\vartheta_{t}(a):=a \odot \mathrm{id}_{t}$ on $\mathcal{B}^{a}(E)$, the free flow of free index $F$. We immediately read of from this factorization that the product system of $\vartheta$ is

$$
\mathcal{F}(F)^{\odot}=\left(\omega \mathcal{B} \oplus\left(E_{[0, t)} \odot \mathcal{F}(E)\right)\right)_{t \in \mathbb{R}_{+}} .
$$

When $F=K$ is a (separable) Hilbert space, Fowler [Fow95] showed that $\vartheta$ is cocycle conjugate to a CCR flow of index $\infty$, independently of the dimension of $K$. In Skeide [Ske06b] we showed for arbitrary Hilbert $\mathcal{B}-\mathcal{B}$-bimodules $F$ that $\vartheta$, actually, is a CCR-flow of index $F \odot \mathcal{F}(E)$.

However, in [Ske06b] we also mentioned that the free flow on $\mathcal{F}(E)$ can be suitably interpreted in terms of free product systems. In our talk we give the first concise definition of free product systems, we discuss their relation with $E_{0}$-semigroups (all noises; see the introduction), and we discuss how spatial tensor product systems can be blown up to obtain free product systems. We raise several natural questions that have to be answered. 
Let us recall that the free product of two Hilbert $\mathcal{B}$ - $\mathcal{B}$-bimodules $E_{i}$ with respect to unital central reference vectors $\omega_{i} \in F_{i}$ is

$$
E_{1} \circledast E_{2}:=\bigoplus_{n \in \mathbb{N}_{0}} \bigoplus_{\varepsilon \in A_{n}(\{1,2\})} E_{\varepsilon}=\bigoplus_{n \in \mathbb{N}_{0}} \bigoplus_{\varepsilon \in A_{n}(\{1,2\})} \omega_{\varepsilon_{1}}^{\perp} \odot \ldots \odot \omega_{\varepsilon_{n}}^{\perp} \text {, where }
$$

$A_{n}(S):=\left\{\varepsilon \in S^{n}: \varepsilon_{i} \neq \varepsilon_{i+1}\right\}$ and $E_{()}:=\omega \mathcal{B}$ with $A_{0}(S):=\{()\}$. Note that $E_{1} \circledast E_{2} \supset \omega \mathcal{B} \oplus \omega_{i}^{\perp}=E_{i}$. Using this, we find

$$
E_{1} \circledast E_{2}=E_{1} \odot\left(\omega \mathcal{B} \oplus \bigoplus_{n \in \mathbb{N}} \bigoplus_{\varepsilon \in A_{n}(\{1,2\}), \varepsilon_{1} \neq 1} E_{\varepsilon}\right),
$$

so that there is a unital embedding of $\mathcal{B}^{a}\left(E_{1}\right)$ as $\mathcal{B}^{a}\left(E_{1}\right) \odot$ id into $\mathcal{B}^{a}\left(E_{1} \circledast E_{2}\right)$.

Apart from the above mentioned tensor factorization, the full Fock module also satisfies the factorization

$$
\mathcal{F}\left(F_{1} \oplus F_{2}\right)=\mathcal{F}\left(F_{1}\right) \circledast \mathcal{F}\left(F_{2}\right)
$$

with respect to the respective vacua. If we put $\mathcal{F}_{t}(F):=\mathcal{F}\left(E_{[0, t)}\right)$, then the family $\mathcal{F} \circledast(F):=\left(\mathcal{F}_{t}(F)\right)_{t \in \mathbb{R}_{+}}$forms a free product system in the sense of the following definition sketched already in [Ske06b]:

Definition. Algebraically, a free product system is a family of Hilbert $\mathcal{B}-\mathcal{B}-$ bimodules $E^{\circledast}=\left(E_{t}\right)_{t \in \mathbb{S}}$ with a family $\omega^{\circledast}=\left(\omega_{t}\right)_{t \in \mathbb{S}}$ of central unit vectors $\omega_{t} \in E_{t}$ and bilinear unitaries

$$
u_{s, t}:\left(E_{s}, \omega_{s}\right) \circledast\left(E_{t}, \omega_{t}\right) \longrightarrow\left(E_{s+t}, \omega_{s+t}\right)
$$

that compose associatively, and $E_{0}=\omega_{0} \mathcal{B}$ such that $u_{0, t}, u_{t, 0}$ are the canonical identifications.

Technical conditions (continuity, measurability) on the bundle structure may be put as in in the tensor product case. Notice that $E_{s} \odot E_{t} \subset E_{s} \circledast E_{t}$, so that it makes also sense to require that the product of continuous (measurable) sections is continuous (measurable) in any version we prefer.

Since $E_{t} \cong \omega_{s} \circledast E_{t} \subset E_{s} \circledast E_{t} \cong E_{s+t}$, we obtain an inductive limit $E_{\infty} \ni \omega$, which is a Hilbert $\mathcal{B}-\mathcal{B}$-bimodule and has a unital central reference vector $\omega$.

Theorem. The inductive limit comes with a free left dilation

$$
v_{t}: E_{\infty} \circledast E_{t} \longrightarrow E_{\infty}
$$

that iterates associatively with the free product system structure. The unital embeddings $\mathcal{B}^{a}\left(E_{\infty}\right) \rightarrow \mathcal{B}^{a}\left(E_{\infty} \circledast E_{t}\right) \cong \mathcal{B}^{a}\left(E_{\infty}\right)$ induce, therfore, an $E_{0}$-semigroup $\vartheta$ on $\mathcal{B}^{a}\left(E_{\infty}\right)$.

Since $E_{\infty} \circledast E_{t}=E_{\infty} \odot\left(\omega \mathcal{B} \oplus \bigoplus_{n \in \mathbb{N}} \bigoplus_{\varepsilon \in A_{n}(\{\infty, t\}), \varepsilon_{1} \neq \infty} E_{\varepsilon}\right)$, the (tensor!) product system of $\vartheta$ is

$$
\omega \mathcal{B} \oplus \bigoplus_{n \in \mathbb{N}} \bigoplus_{\substack{\varepsilon \in A_{n}(\{\infty, t\}) \\ \varepsilon_{1} \neq \infty}} E_{\varepsilon} .
$$


In the case of the free product system of the free flow on a full Fock module we get back the free flow we started with.

An open problem is to characterize all $E_{0}$-semigroups (necessarily noises) that arise in this way.

Unlike the $E_{0}$-semigroups described by their tensor product system, the free flows come along with unital embeddings of $\mathcal{B}^{a}\left(E_{t}\right)$ into $\mathcal{B}^{a}\left(E_{\infty}\right)$ arising from the identification $E_{\infty}=E_{\infty} \circledast E_{t}=E_{t} \circledast E_{\infty}$. (The tensor product system description $E=E \odot E_{t}$ allows only nonunital embeddings as $(\omega \odot$ id $) \mathcal{B}^{a}\left(E_{t}\right)\left(\omega^{\circledast} \odot\right.$ id); see the introduction.) Moreover, these subalgebras generate a filtration that is conditionally free independent in the vacuum expectation $\langle\omega, \bullet \omega\rangle$. (The filtration from the nonunital embedding in the tensor case is only conditionally monotone independent.)

An open problem is to find out what it means for a cocycle to be adapted to the free filtration. We hope that in the Fock situation a solution of that problem might enable us to write down adapted unitary cocycles directly from the (wellknown) units of the tensor product system picture.

We mentioned already that in a free product system we have $E_{s} \odot E_{t} \subset E_{s+t}$. (Contrary to what has been considered in Bhat's talk on Page 34 and in Shalit's talk on Page 27, respectively, this would not be an inclusion system and a subproduct system, respectively, but rather a superproduct system. This is also closely related to a situation described in Köstler's talk on Page 29.) Clearly, the intersection $\bigcap_{\mathfrak{t}} E_{t_{n}} \odot \ldots \odot E_{t_{1}}$ over all $\mathfrak{t}=\left(t_{n}, \ldots, t_{1}\right)$ with $t_{1}+\ldots+t_{n}=t$ is the biggest tensor product subsystem of the free product system. An interesting open problem is, whether this subsystem generates $E^{\circledast}$ as a free product system. In a minute we see that in the Fock situation the answer is affirmative. But, it is not to be excluded that, in the worst case, the intersection consists only of the vacuum sector $\omega_{t} \mathcal{B}$.

Let $\left(E^{\odot}, \omega^{\odot}\right)$ be a spatial tensor product system, see the introduction . For each $\mathfrak{t}=\left(t_{n}, \ldots, t_{1}\right)$ with $t_{1}+\ldots+t_{n}=t$ define

$$
\mathcal{E}_{\mathfrak{t}}:=E_{t_{n}} \circledast \ldots \circledast E_{t_{1}}
$$

By the inclusion $E_{t}=E_{t_{n}} \odot \ldots \odot E_{t_{1}} \subset \mathcal{E}_{\mathfrak{t}}$ and since free products respect inclusions, we obtain an inductive limit $\varepsilon_{t}$ of $\varepsilon_{\mathfrak{t}}$ over $\mathfrak{t}$.

Theorem. The $\varepsilon_{t}$ form a free product system $\varepsilon^{\circledast}$. It contains $E^{\odot}$ as a tensor product subsystem and is generated by $E^{\odot}$ as a free product system.

An open problem is, if we get back $E^{\odot}$ by the intersection procedure as described before. In general, we ask when a free product system be obtained in that way from a spatial tensor product subsystem. 


\section{Isometric Dilations of Representations of Product Systems of $C^{*}$-Correspondences over $\mathbb{N}_{0}^{k}$}

\section{ADAM SKALSKI}

Classical multi-dimensional dilation theory $([\mathrm{SzF}])$ for Hilbert space operators is concerned with dilating tuples of contractions to tuples of isometries or unitaries, preserving some specific properties of the original family. Celebrated examples of S. Parrott, N. Varopoulos and others show that commuting isometric dilations of more than two commuting contractions need not exist. In general we cannot expect a characterisation of these tuples for which the commuting dilation exists, however if one requests a specific form of the dilation then precise answers can be obtained. In particular the existence of so-called regular or ${ }^{*}$-regular dilations (i.e. dilations satisfying additional conditions with respect to products of the original contractions and their adjoints, see [Bre], [Tim]) can be detected via simple conditions corresponding to positive-definiteness of certain operator-valued functions associated with the initial tuple.

In this talk we discuss analogous results for dilations of representations of product systems of representations of product systems of $C^{*}$-correspondences over $\mathbb{N}_{0}^{k}$. For us a $C^{*}$-correspondence $E$ over a $C^{*}$-algebra $\mathrm{A}$ is a $C^{*}$-Hilbert module (a right module over A with A-valued scalar product) equipped additionally with the structure of a left module over A. Formally: there is a nondegenerate $*$-homomorphism $\phi: \mathrm{A} \rightarrow \mathcal{L}(E)$.

Definition. A product system of $C^{*}$-correspondences over $\mathbb{N}_{0}^{k}$, denoted by $\mathbb{E}$, is a family of $k C^{*}$-correspondences $\left\{E_{1}, \ldots, E_{k}\right\}$ over a $C^{*}$-algebra $\mathrm{A}$ together with the unitary isomorphisms $t_{i, j}: E_{i} \otimes E_{j} \rightarrow E_{j} \otimes E_{i}(i>j)$ satisfying a natural associativity condition.

For the purpose of this talk we think of each $E_{i}$ describing the type of the $i$-th element of the tuple we intend to dilate (a contraction, a row contraction, a family of contractions associated to a graph) and of $t_{i, j}$ as means of encoding the commutation relations between different elements of the tuple.

Definitions of completely contractive representations of $\mathbb{E}$ and their isometric dilations can be found in [Sol] or [Sk]. In [Sol] Solel characterised the existence of regular isometric dilation of a given representation via Brehmer-type conditions. Here we focus on ${ }^{*}$-regular dilations. Contrary to the classical context of dilating commuting tuples of contractions regular dilations cannot be straightforwardly transformed into ${ }^{*}$-regular ones. We still however have the following result:

Theorem. A minimal isometric dilation $\vec{V}$ of a representation $\vec{T}$ of $\mathbb{E}$ is ${ }^{*}$-regular if and only if it is doubly commuting.

Under a certain technical condition on the product system $\mathbb{E}$ and the assumption that a given representation $\vec{T}$ of $\mathbb{E}$ on a Hilbert space $\mathrm{H}$ satisfies a so-called Popescu condition (or condition 'P'), ${ }^{*}$-regular isometric dilation of $\vec{T}$ can be constructed via so-called generalised Poisson transform. Generalised Poisson transform is a completely positive map $R_{\vec{T}}: \mathcal{T}_{\mathbb{E}} \rightarrow B(\mathrm{H})$, where $\mathcal{T}_{\mathbb{E}}$ is the Toeplitz-type algebra 
associated with $\mathbb{E}$. The map $R_{\vec{T}}$ was earlier studied in a simpler context for example in [Pop]. Sufficient conditions for the construction of $R_{\vec{T}}$ are summarised in the following theorem.

Theorem. Let $\mathbb{E}$ have a normal ordering property and let $\vec{T}$ be a representation of $\mathbb{E}$ on $\mathrm{H}$ satisfying the Popescu condition. Then there exists a unique continuous linear map $R_{\vec{T}}: \mathcal{T}_{\mathbb{E}} \rightarrow B(\mathrm{H})$ satisfying

$$
R_{\vec{T}}\left(L_{e} L_{f}^{*}\right)=T(n)(e)(T(m)(f))^{*}, \quad n, m \in b n_{0}^{k}, e \in \mathbb{E}(n), f \in \mathbb{E}(m) .
$$

The map $R_{\vec{T}}$ is completely positive and contractive, unital if $\mathcal{T}_{\mathbb{E}}$ is unital.

The Stinespring dilation for $R_{\vec{T}}$ provides in a natural way an isometric dilation for $\vec{T}$.

In the second part of the talk we present applications of the above results to families of contractions associated to a given higher-rank graph $\Lambda$ ([KuPa], [Rae]). There is a natural way of associating to $\Lambda$ a product system $\mathbb{E}(\Lambda)([\mathrm{RaS}])$ and it can be showed that $\mathbb{E}(\Lambda)$ has the normal ordering property if and only if $\Lambda$ is finitely aligned ([Rae $]$ ) and if and only if $\mathbb{E}(\Lambda)$ is compactly aligned ([Fow $]$ ). We have the following result:

Theorem. There is a 1-1 correspondence between (completely contractive) representations of $\mathbb{E}(\Lambda)$ on a Hilbert space $\mathrm{H}$ and $\Lambda$-contractions in $B(\mathrm{H})$. The representation is isometric if and only if the corresponding $\Lambda$-contraction forms a Toeplitz family, isometric and doubly commuting if and only if the corresponding $\Lambda$-contraction forms a Toeplitz-Cuntz-Krieger family.

The above correspondence can be used to transform our and Solel's results on dilation of representations of product systems to the context of dilating $\Lambda$ contractions. As an example we present the following theorem, first proved in [SkZ]:

Theorem. Let $\Lambda$ be finitely aligned and let $\mathcal{V}$ be a $\Lambda$-contraction on a Hilbert space $\mathrm{H}$ which satisfies the Popescu condition. Then there exists a Hilbert space $\mathrm{K} \supset \mathrm{H}$ and a $\Lambda$-contraction $\mathcal{W}$ on $\mathrm{K}$ consisting of partial isometries forming a Toeplitz-Cuntz-Krieger family such that

$$
\left.W_{\lambda}^{*}\right|_{H}=V_{\lambda}^{*}, \quad \lambda \in \Lambda .
$$

One may assume that $\mathrm{K}=\overline{\operatorname{Lin}}\left\{W_{\lambda} \mathrm{H}: \lambda \in \Lambda\right\}$; under this assumption the family $\mathcal{W}$ is unique up to unitary equivalence.

Most of the results presented in the talk can be found in $[\mathrm{Sk}]$.

\section{REFERENCES}

[Bre] S. Brehmer, Über vetauschbare Kontraktionen des Hilbertschen Raumes, Acta Sci. Math. Szeged 22 (1961), 106-111.

[Fow] N. Fowler, Discrete product systems of Hilbert bimodules, Pacific J. Math. 204 (2002), 335-375. 
[KuPa] A. Kumjian and D. Pask, Higher rank graph $C^{*}$-algebras, New York J. Math. 6 (2000), $1-20$.

[Pop] G. Popescu, Poisson transforms on some $C^{*}$-algebras generated by isometries, $J$. Funct. Anal. 161 (1999), no.1, 27-61.

[Rae] I. Raeburn, "Graph $C^{*}$-algebras", CBMS Regional Conference Series in Mathematics, 103, Providence, RI, 2005

[RaS] I. Raeburn and A.Sims, Product systems of graphs and the Toeplitz algebras of higherrank graphs, J. Operator Theory $\mathbf{5 3}$ (2005), no. 2, 399-429.

[Sk] A. Skalski, On isometric dilations of product systems of $C^{*}$-correspondences and applications to families of contractions associated to higher-rank graphs, Indiana Univ. J. Math., to appear.

[SkZ] A. Skalski and J. Zacharias, Poisson transform for higher-rank graph algebras and its applications, J. Operator Theory, to appear.

[Sol] B. Solel, Regular dilations of representations of product systems, Math. Proc. Royal Irish Soc. 108 (2008), no. 1, 89-110.

[SzF] B. Sz.-Nagy and C. Foias, "Harmonic analysis of operators on Hilbert space", North Holland, Amsterdam, 1970.

[Tim] D. Timotin, Regular dilations and models for multicontractions, Indiana Univ. Math. J. 47 (1998), no. 2, 593-618.

\section{An Approach to the Mathematical Theory of Pressure Broadening of Spectral Lines}

\section{WILHELM VON WALDENFELS}

One of the three "natural" independences of quantum probability is called "Boolean independence" and the authors cite my old paper [1]. As this paper is not well known, I was asked to present it at the workshop.

Pressure broadening is a stochastic Stark effect: due to fluctuating electrical fields a spectral line coming from a plasma appears broadened. One considers an atom and the transition between two energy levels, which might be degenerate. The electrical field is generated by the electrons passing by. The electrons are assumed to be independent, they form a Poisson point process, they move on straight lines with constant velocity and their directions are uniformly distributed on the unit sphere. The mathematical problem comes from the fact, that the interactions with the atom overlap in time. The time of nearest approach to the atom is called impact time, the vector from the atom to the electron at the nearest approach is called impact parameter. If the distance between the impact times is very big, then the interactions are independent and the overlapping can be neglected. These considerations suggest a cluster expansion.

It is not the point, to present here the main features and the result of the theory. Instead I want to describe the principle of the cluster expansion and a typical example.

We consider intervals of the form

$$
A=(k, k+1, \cdots, l-1, l)
$$

in $\mathbb{N}$. We say that $A_{1}, \cdots, A_{K}$ form an interval partition of $A$ and write

$$
A=A_{1} \circ A_{2} \circ \cdots \circ A_{K}
$$


if

$$
\begin{aligned}
A_{1} & =\left(k, \cdots, l_{1}\right), \\
A_{2} & =\left(l_{1}+1, \cdots, l_{2}\right), \\
& \vdots \\
A_{K} & =\left(l_{K-1}+1, \cdots, l_{K}=l\right) .
\end{aligned}
$$

We form the free algebra $\mathfrak{A}$ generated of all the intervals of the form $A$ and define the cumulants recursively by the formula

$$
A=\sum_{A_{1} \circ \cdots \circ A_{K}=A}\left[A_{1}\right] \cdots\left[A_{K}\right] .
$$

That implies

$$
[A]=\sum_{A_{1} \circ \cdots \circ A_{K}=A}(-1)^{K+1} A_{1} \cdots A_{K} .
$$

We define the splitting operator

$$
P_{m}(k, \cdots, l)= \begin{cases}(k, \cdots, m)(m+1, \cdots, l) & \text { for } k \leq m<l \\ (k, \cdots, l) & \text { otherwise }\end{cases}
$$

and extend it to an homomorphism $\mathfrak{A} \rightarrow \mathfrak{A}$. We obtain

$$
P_{m}[k, \cdots, l]=\left\{\begin{array}{ll}
0 & \text { for } k \leq m<l \\
{[k, \cdots, l]} & \text { otherwise }
\end{array} .\right.
$$

These equations are special cases of relations holding in partially ordered sets [2], related to the names $\zeta$-function and Moebius function.

Assume, that the electrical field strength due to an electron with impact time 0 and impact parameter $p$ and direction $v$ is given by $\varphi(\zeta)(t)$ with $\zeta=(p, v)$. We truncate and assume, that $\varphi(\zeta)(t)=0$ for $|t|>\tau / 2, p>\varrho$. The Hilbert space is the line space $\mathfrak{H}$, the tensor product of the two eigenspaces of the Hamiltonian between which the transition takes place. The effect of electrons with impact parameters $\zeta_{k}, \cdots, \zeta_{l}$ and impact times $t_{k}<\cdots<t_{l}$ is at time $t$

$$
h\left(\varphi\left(\zeta_{k}\right)\left(t-t_{k}\right)+\cdots+\varphi\left(\zeta_{l}\right)\left(t-t_{l}\right)\right)
$$

where $h$ is a function from $\mathbb{R}^{3}$ into the space of hermitian operators in the finite dimensional line space $\mathfrak{H}$ with the property, that $h(0)=0$, e.g. a linear function or a quadratic function. If $k \leq m<l$ and $t_{m+1}-t_{m}>\tau$ then

$$
\begin{array}{r}
h\left(\varphi\left(\zeta_{k}\right)\left(t-t_{k}\right)+\cdots+\varphi\left(\zeta_{l}\right)\left(t-t_{l}\right)\right)=h\left(\varphi\left(\zeta_{k}\right)\left(t-t_{k}\right)+\cdots+\varphi\left(\zeta_{m}\right)\left(t-t_{m}\right)\right) \\
\left.+h\left(\varphi\left(\zeta_{m+1}\right)\left(t-t_{m+1}\right)\right)+\cdots+\varphi\left(\zeta_{l}\right)\left(t-t_{l}\right)\right) .
\end{array}
$$

Due to the truncation of the impact parameter $p$ there exists a constant $c$ such that the difference between two impact times is distributed with respect to an exponential law with parameter $c$, i.e.

$$
\text { probability }\left\{t_{k+1}-t_{k} \in(t, t+d t)\right\}=c e^{-c t} d t \text {. }
$$


The time differences are independent and the $\zeta_{k}$ are independent identical distributed on $\mathbb{R}^{3} \times \mathbb{S}^{2}$.

We want now to discuss a part of the problem, which is relevant for the line center. Assume a sequence of independent random variables $u_{1}, u_{2}, \cdots$ exponentially distributed with parameter $c$. Denote by $\Theta$ the right shift, so $(\Theta(s) \varphi(\zeta))(s)=$ $\varphi(\zeta)(t-s)$. Define for $A=(k, \cdots, l)$

$$
\begin{aligned}
& U(A)=u_{k}+\cdots+u_{l} \\
& \Phi(A)=h\left(\varphi\left(\zeta_{k}\right)+\Theta\left(u_{k}\right) \varphi\left(\zeta_{k+1}\right)+\cdots+\Theta\left(u_{k}+\cdots+u_{l-1}\right) \varphi\left(\zeta_{l}\right)\right) \\
& S(A)=\prod_{-\infty}^{+\infty}(1+i \Phi(A)(t) d t) \\
& \alpha(A)=\delta_{U(A)} S(A)
\end{aligned}
$$

where $\delta_{U(A)}$ is the point measure in the point $U(A)$ and the product integral $\prod$ has to be read from the right to the left with increasing time. If $u_{m}>\tau$ for $k \leq m<k$, denote $A_{1}=(k, \cdots, m), A_{2}=(m+1, \cdots, l)$ and obtain

$$
\begin{aligned}
& U(A)=U\left(A_{1}\right)+U\left(A_{2}\right) \\
& \Phi(A)=\Phi\left(A_{1}\right)+\Theta\left(U\left(A_{1}\right)\right) \Phi\left(A_{2}\right) \\
& S(A)=S\left(A_{2}\right) S\left(A_{1}\right) \\
& \alpha(A)=\alpha\left(A_{2}\right) * \alpha\left(A_{1}\right),
\end{aligned}
$$

where $*$ denotes the usual convolution. Extending $\alpha$ to the algebra $\mathfrak{A}$ one gets in this case $\alpha[A]=0$. After some straight forward calculations one obtains

$$
\begin{aligned}
& \mathbb{E}(A(1)+A(1,2)+A(1,2,3)+\cdots)=\frac{\alpha}{\delta-\alpha}=\alpha *(\delta-\alpha)^{*-1} \\
& \alpha=\alpha_{1}+\alpha_{2}+\alpha_{3}+\cdots \\
& \alpha_{k}=\mathbb{E} \alpha[1,2, \cdots, k] .
\end{aligned}
$$

As $\alpha[1,2, \cdots, k]=0$ if $u_{i}>\tau$ for any of the $u_{i}, i=1, \cdots, k-1$ the $L_{1^{-}}$norm of $\alpha_{k}$ is $\leq 2^{k-1}\left(1-e^{-c \tau}\right)^{k-1}$ and the sum defining $\alpha$ converges for $c \tau \leq \ln 2$.

\section{REFERENCES}

[1] W.von Waldenfels. An Approach to the Theory of Pressure Broadening of Spectral Lines. Probability and Information Theory II. M.Behara,K. Krickeberg (eds) Lecture Notes in Mathematics, 296. Springer 1973.

[2] W.von Waldenfels. Interval Partitions and Pair Interactions. Seminaire de Probabilites,Universite de Strasbourg XI. P.A. Meyer (ed) Lecture Notes in Mathematics 465, Springer 1975. 


\title{
Transformation of Quantum Lévy Processes Michael SchürmanN
}

Let $(\mathcal{B}, \Delta, \delta)$ and $(\mathcal{C}, \Lambda, \lambda)$ be two dual semigroups in the sense of $\mathrm{D}$. Voiculescu [Voi] with comultiplications $\Delta, \Lambda$ and counits $\delta, \lambda$. Suppose that there is also given a *algebra homomorphism $\kappa: \mathcal{C} \rightarrow \mathcal{B}$ such that

$$
\delta \circ \kappa=\lambda \text {. }
$$

Then, if $\psi$ is the generator of a quantum Lévy process (QLP) $j_{s t}$ on $\mathcal{B}$ in the sense of [BGSch2], $\psi \circ \kappa$ is the generator of a QLP $k_{s t}$ on $\mathcal{C}$. The QLP $k_{s t}$ can be constructed from the QLP $j_{s t}$ by a 'convolution product integral'.

Applying this construction to the dual semigroups $\left(\mathrm{T}\left(\mathcal{B}_{0}\right), \mathrm{T}\left(\Delta_{0}\right), \mathrm{T}(0)\right)$ and $\left(\mathrm{T}\left(\mathcal{B}_{0}\right)\right.$, $\left.\mathrm{T}\left(\iota_{1}+\iota_{2}\right), \mathrm{T}(0)\right)$, it can be seen that $j_{s t}$ can be realized on a Fock space (the type of which depends on the chosen independence) and that the vacuum vector is cyclic for this realization.

\section{REFERENCES}

[BGSch1] Ben Ghorbal, A., Schürmann, M.: Non-commutative notions of stochastic independence. Math. Proc. Camb. Phil. Soc. 133, 531-561 (2002)

[BGSch2] Ben Ghorbal, A., Schürmann, M.: Quantum Lévy processes on dual groups. Math. Z. 251, 147-165 (2005)

[Fra1] Franz, U.: Lévy processes on quantum groups and dual groups. In: Quantum Independent Increment Processes II, Lect. Notes Math., vol. 1866, Springer 2006

[Fra3] Franz, U.: Unification of boolean, monotone, anti-monotone and tensor independence and Lévy processes. Math. Z. 243, 779-816 (2003)

[Mur1] Muraki, N.: The five independences as quasi-universal products. IDAQP 5, 113-134 (2002)

[Mur2] Muraki, N.: The five independences as natural products. IDAQP 6, 337-371 (2003)

[Sch] Schürmann, M.: Direct sums of tensor products and non-commutative independence. J. Funct. Anal. 133, 1-9 (1995)

[SchSkV] M. Schürmann, M. Skeide, S. Volkwardt: Transformations of Lévy processes. arXiv:0712.3504v2

[Sp2] Speicher, R.: On universal products. Fields Inst. Commun. 12, 257-266 (1997)

[SchVo] M. Schürman, S. Voß: Positivity of free convolution semigroups. Proceedings of the conference Quantum Probability Related Topics, Guanajuato, Mexico 2007

[Voi] D. Voiculescu: Dual algebraic structures on operator algebras related to free products. J. Operator Theory 17, 85-98 (1987)

[vW] W. von Waldenfels: Ito solution of the linear quantum stochastic differential equation describing light emission and absorption. In: Accardi, L., Frigerio, A., Gorini, V. (eds.) Quantum probability and applications to the theory of irreversible processes. Proceedings, Villa Mondragone 1982, Lect. Notes Math., vol. 1055, Springer 1984

\section{Theory of Integration in Izumi-Srinivasan Generalized CCR Flows}

\author{
KALYAN B. SINHA
}

(joint work with R. Srinivasan) 
This is an account of a preliminary attempt to formulate the concepts of adapted ness and integration in the context of the theory of generalized CCR flow, constructed by Izumi and Srinivasan [1] as examples of a type III product systems.

In this set-up, we shall work in $H=L^{2}\left(\mathbb{R}_{+}\right)_{\mathbb{R}}$ (for our purposes, the multiplicity space $\mathrm{K}$ is not so important) and we are given two $C_{o}$-semi groups $T=\left(T_{t}\right)_{t>0}$, $S=\left(S_{t}\right)_{t \geq 0}$ in $\mathrm{H}$ (of which $\mathrm{S}$ is the right shift in $L^{2}\left(\mathbb{R}_{+}\right)_{\mathbb{R}}$, i.e $\left(S_{t} f\right)(x)=f(x-t)$ for $x>t,=0$ for $x<=t$ ) satisfying,

(i) $T_{t}^{*} S_{t}=I$ and

$$
\text { (ii) } T_{t}-S_{t} \epsilon \mathbf{B}_{2}(H) \text { forall } t \geq 0 \text {. }
$$

Then the flow $\varphi$ defined by:

$$
\varphi_{t}(W(f))=W\left(S_{t} f_{R}+i T_{t} f_{I}\right)
$$

with $f=f_{R}+i f_{I}$, gives a generalized CCR flow which extends to a $E_{o}$-semi group on $\mathbf{B}\left(\Gamma\left(H^{\mathbb{C}}\right)\right)$.

Thus there are two different "shifts"-one for the real part and the other for the imaginary part; hence, we can expect that the definition of 'past' and 'future' will be different for real and imaginary parts, unlike the traditional HudsonParthasarathy theory.

Set $p_{t}=I-S_{t} T_{t}^{*}, t \geq 0$ Then one has

LEMMA 1: (i) $p_{t}$ is a real idempotent, not necessarily self-adjoint,

(ii) Range $\left(p_{t}\right)=\operatorname{ker}\left(T_{t}^{*}\right)$ and Range $\left(p_{t}^{*}\right)=\operatorname{ker}\left(S_{t}^{*}\right)$,

(iii) $p_{t+s}=p_{s}+S_{s} p_{t} T_{t}^{*}$ (and we write $p_{t}^{s}=S_{s} p_{t-s} T_{s}^{*}$, for $0 \leq s<t$ ),

(iv) $p_{s} p_{t}^{s}=p_{t}^{s} p_{s}=0$ for $0 \leq s<t$,

(v) $p_{s} p_{t}=p_{t} p_{s}=p_{s \wedge t}$

(vi) $p_{t}-p_{t}^{*}=2 i \operatorname{Im}\left(T_{t}-S_{t}\right) S_{t}^{*} \epsilon B_{2}(H)$ and $p_{t}=p_{t}^{*}$ if and only if $T_{t}=S_{t}$.

As has been argued in [1] we can assume without loss of generality that $p_{\infty}=I$. We write $f_{t}=p_{t} f_{R}+i p_{t}^{*} f_{I}$ and $f^{t}=p^{t} f_{R}+i p^{t^{*}} f_{I}$

$$
\text { where } p^{t}=I-p_{t}=S_{t} T_{t}^{*} \text {. }
$$

so that $f=f_{t}+f^{t}$ in $H^{\mathbb{C}}$

The Weyl operators behave well with respect to this splitting.

LEMMA 2:(i) $W(f)=W\left(f_{t}\right) W\left(f^{t}\right)=W\left(f^{t}\right) W\left(f_{t}\right)$ for $f \epsilon H^{\mathbb{C}}$ and every $t \geq 0$.

(ii) More generally, if we set $f_{t}^{s}=p_{t}^{s} f_{r}+i p_{t}^{s^{*}} f_{I}, W\left(f_{t}^{s}\right)$ and $W\left(f_{t^{\prime}}^{s^{\prime}}\right)$ commute if

$$
[s, t] \cap\left[s^{\prime}, t^{\prime}\right]=\emptyset .
$$

If we now set $B_{s, t}=$ the von Neumann algebra generated by $\left\{W\left(f_{t}^{s}\right) \mid f \in L^{2}\left(\mathbb{R}_{+}\right)_{\mathbb{C}}\right\}$, then it is clear that $B_{t}^{\prime}=B^{t}$ where we have set $B_{t}=B_{[o, t]}$ and $B^{t}=B_{[t, \infty]}$. 
Because of the structure given above, it seems that the integrators here would be the "position" and "momentum" operators rather than the annihilation and creation operators in $\Gamma\left(H^{\mathbb{C}}\right)$. We define $P\left(f_{R}\right)$ and $Q\left(f_{I}\right)$ as

$W\left(\lambda f_{R}\right)=e^{i \lambda P\left(f_{R}\right)}$ and $W\left(i \lambda f_{I}\right)=e^{i \lambda Q\left(f_{I}\right)}$ and note the commutation relation:

$$
\left[P\left(f_{R}\right), Q\left(g_{I}\right)\right]=2 i\left\langle f_{R}, g_{I}\right\rangle,
$$

with the other P-P and Q-Q commutators vanishing. With this definition, it is clear that both $P\left(p_{t}^{s} f_{R}\right)$ and $Q\left(p_{t}^{s^{*}} f_{I}\right)$ commute with $B\left[s^{\prime}, t^{\prime}\right]$ whenever $[s, t] \cap\left[s^{\prime}, t^{\prime}\right]=\emptyset$

We say that a family $X \equiv\left\{X_{t}, t \geq 0\right\}$ of bounded operators in $\Gamma\left(H^{\mathbb{C}}\right)$ adapted with respect to the filtration $B_{t}$ if $X_{t} \epsilon B_{t} \forall t \geq o$ and it is said to be regular if $t \rightarrow X_{t}$ is strongly continuous.

With these concepts, one can next define the integrals of an adapted process with respect to the integrators as above. However, the convenient expressions are yet to be derived and suitable "quantum Ito formula" has to emerge.

\section{REFERENCES}

[1] M. Izumi and R. Srinivasan, Generalized CCR-flows, Comm. Math. Phys. 281 (2), 529-571, 2008.

\section{Some Comments on Product Systems and Markov Processes LUIGI ACCARDI}

Following our suggestion, in the conference talks $[1,2]$ it has been pointed out how the product system of Hilbert modules associated with the Markov semigroup of a classical stationary Markov process over $\mathbf{R}_{+}$can be obtained directly from the process.

In this talk, as a motivation, we first describe a version for the nonstationary case, and then generalize this to Markov fields. The field version requires a concise definition of a family of admissible subsets of $\mathbf{R}^{d}$ which allows, in some sense, to resemble that some subsets are "further outside" than others. After giving such a definition, we obtain a far reaching generalization of product systems indexed by subsets of $\mathbf{R}^{d}$ with an interior and an exterior boundary which consists of Hilbert bimodules over the two local algebras related to the two boundaries.

In the end, we make some considerations about time reflection. We suspect a deep interelation of time reflection with the commutant of von Neumann bimodules, and pose it as an open problem to investigate this connection. 


\section{REFERENCES}

[1] M. Skeide. Independence and product systems. In S. Albeverio, Z.-M. Ma, and M. Röckner, editors, Recent developments in stochastic analysis and related topics, pages 420-438. World Scientific, 2004. (arXiv: math.OA/0308245).

[2] M. Skeide. Lévy processes and tensor product systems of Hilbert modules. In M. Schürmann and U. Franz, editors, Quantum Probability and Infinite Dimensional Analysis - From Foundations to Applications, number XVIII in Quantum Probability and White Noise Analysis, pages 492-503. World Scientific, 2005.

\section{Open Problems}

In this section we reproduce several open problems that were discussed at the Mini-Workshop.

\section{B.V.R. Bhat: Type of Inductive Limits of FInite-dimensional INCLUSION SYSTEMS}

Let $\left\{E_{t}: t \in \mathbb{R}_{+}\right\}$be a finite-dimensional inclusion systems, i.e. a family of finitedimensional Hilbert spaces with isometries

$$
\beta_{s, t}: E_{s+t}: E_{s+t} \rightarrow E_{s} \otimes E_{t}, \quad, s, t \geq 0,
$$

satisfying the usual assumptions of associativity, etc. Then $\left\{E_{t}: t \in \mathbb{R}_{+}\right\}$can be embedded into a product system $\left\{\hat{E}_{t}: t \in \mathbb{R}_{+}\right\}$.

Question: Is it possible to get product systems of type II or III in this way?

It may be noted that finite dimensional inclusion systems of CP semigroups on $M_{n}$ give rise to type I product systems. For more information about the context of the problem and references see the abstract of the Bhat's talk on page 34 .

\section{Claus KÖstler: Does SPREAdibility IMPLy BRAidability?}

In $[1,2]$, exchangeability, braidability, and spreadibility for sequences of quantum random variables were introduced. It was shown that they imply tail independence. Furthermore, exchangeability implies braidability, and braidability implies spreadability. We have constructed examples that prove that these notions are stronger than tail independence and that spreadability does not imply exchangeability. In view of these results, it remains still open if spreadability implies already braidability.

\section{REFERENCES}

[1] Claus Köstler, A noncommutative extended de Finetti theorem, arXiv:0806.3621, 2008.

[2] Rolf Gohm and Claus Köstler, Noncommutative independence from the braid group $B_{\infty}$, arXiv:0806.3691v2, to appear in Comm. Math. Phys, 2008. 


\section{Volkmar Liebscher: On Quantum IndePendence}

One might argue, that de Finettis Theorem (see talk of Claus Köstler) may serve as a basis to define independence of algebras under certain states.

To make the idea clear, we propose the following definition for two subalgebras $\mathcal{B}, \mathcal{C} \subseteq \mathcal{A}$ in an algebraic probability space $(\mathcal{A}, \varphi)$.

Note that the state $\varphi$ on $\mathcal{A}$ induces a state $\varphi \circ\left(\imath_{\mathcal{B}} \sqcup \imath_{\mathcal{C}}\right)$ on the free product $\mathcal{B} \sqcup_{1} \mathcal{C}$ of the two subalgebras, as a consequence of the universal property of the free product.

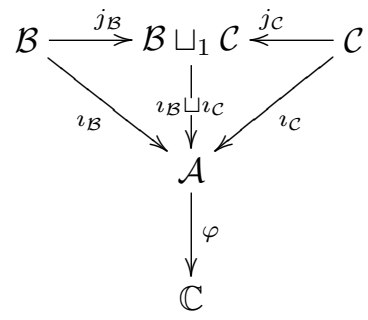

If we embed the subalgebras into two different ("independent") copies of $\mathcal{A}$ inside $\mathcal{A} \sqcup_{1} \mathcal{A} \sqcup_{1} \ldots$, e.g., the first and the second, then any state $\varphi^{\infty}$ on $\mathcal{A} \sqcup_{1} \mathcal{A} \sqcup_{1} \ldots$ induces a state $\varphi^{\infty} \circ\left(\left(j_{1} \circ \imath_{\mathcal{B}}\right) \sqcup\left(j_{2} \circ \imath_{\mathcal{C}}\right)\right)$ on the free product $\mathcal{B} \sqcup_{1} \mathcal{C}$.

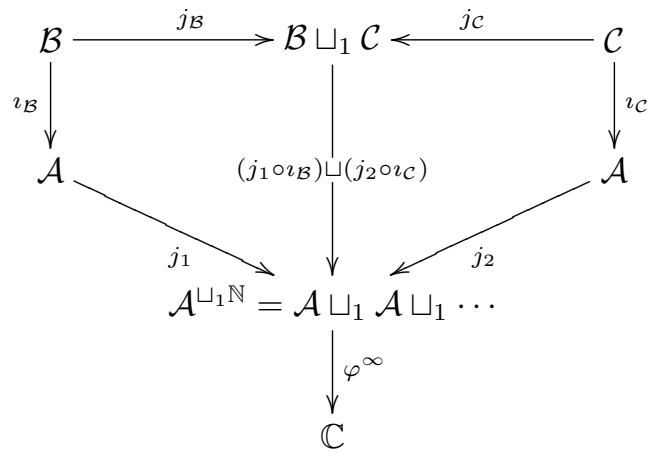

We will call $\mathcal{B}$ and $\mathcal{C}$ independent, if there exists an extremal exchangeable (i.e. $S^{\infty}$-invariant) state on $\mathcal{A} \sqcup_{1} \mathcal{A} \sqcup_{1} \cdots$ such that the two states

$$
\varphi \circ\left(\imath_{\mathcal{B}} \sqcup \imath_{\mathcal{C}}\right) \quad \text { and } \quad \varphi^{\infty} \circ\left(\left(j_{1} \circ \imath_{\mathcal{B}}\right) \sqcup\left(j_{2} \circ \imath_{\mathcal{C}}\right)\right)
$$

coincide. In the classical (i.e. commutative) case, exchangeability and extremality imply that $\varphi^{\infty}$ is a product state (therefore corresponding to a product measure), and we recover the usual notion of independence.

This definition leaves several open questions behind:

(1) We break the dependence on the embedding algebra $\mathcal{A}$ explicitely. The reason is that possibly the embedding $\mathcal{A} \hookrightarrow \mathcal{A}^{\prime}$ need not map the set of extremal $S^{\infty}$-invariant (or exchangeable) states on $\mathcal{A}^{\prime \sqcup_{1} \mathbb{N}}$ onto a superset of the extremal $S^{\infty}$-invariant states on $\mathcal{A}^{\sqcup_{1} \mathbb{N}}$. Is this caution necessary? 
(2) Is the above definition natural, i.e. associative and invariant under algebra morphisms?

(3) Do the 5 natural products obey this definition? The tensor product should do due to [1]. The monotone products are not symmetric, so it seems likely that they are not covered by the above definition. For the free and boolean products, extremality is open but expected.

(4) Are there examples besides the 5 natural products?

(5) What changes, if we substitute the condition "extremal $S^{\infty}$-invariant" by " $S^{\infty}$-invariant with trivial tail field"? In the tensor case, both conditions are equal.

(6) It is not clear to me, whether something changes in the category of von Neumann algebras (only there the tail-field makes full sense) and normal states or $C^{*}$-algebras.

\section{REFERENCES}

[1] E. Stœrmer, Symmetric states of infinite tensor products of $C^{*}$-algebras. J. Funct. Anal. 3 (1969) $48-68$

\section{OrR Shalit: Embedding A Subproduct System in A Product System}

Let $\mathcal{S}$ be a semigroup. A subproduct system (over $\mathcal{S}$ ) is a bundle of $\mathrm{W}^{*}$-correspondences $X=\{X(s)\}_{s \in \mathcal{S}}$ over the $\mathrm{W}^{*}$-algebra $\mathcal{M}:=X(0)$, such that there exist coisometric bimodule maps

$$
U_{s, t}: X(s) \otimes X(t) \rightarrow X(s+t)
$$

that compose associatively, i.e.,

$$
U_{s+t, r}\left(U_{s, t} \otimes I_{X(r)}\right)=U_{s, t+r}\left(I_{X(s)} \otimes U_{t, r}\right) .
$$

This notion has been formalized in [8], but it orginates from Arveson's works [1] and [2].

A subproduct system is called a product system when each map $U_{s, t}$ is a unitary. Products systems of Hilbert spaces (over the semigroup $\mathbb{R}_{+}$) have been introduced in [1] in relation to noncommutative dynamics. See [3] for a detailed account of the theory of product systems of Hilbert spaces, or [9] for a survey of product systems of Hilbert $\mathrm{C}^{*}$-correspondences (over the semigroup $\mathbb{R}_{+}$). Product systems over other semigroups were probably first studied by Fowler, see [5].

Definition 1. Let $X$ and $Y$ be subproduct systems of $\mathcal{M}$ correspondences $(\mathcal{M}$ a $\mathrm{W}^{*}$-algebra) over the same semigroup $\mathcal{S}$. Denote by $U_{s, t}^{X}$ and $U_{s, t}^{Y}$ the coisometric maps that make $X$ and $Y$, respectively, into subproduct systems. $X$ is said to be a subproduct subsystem of $Y$ (or simply a subsystem of $Y$ for short) if for all $s \in \mathcal{S}$ the space $X(s)$ is a closed subspace of $Y(s)$, and if the orthogonal projections $p_{s}: Y(s) \rightarrow X(s)$ are bimodule maps that satisfy

$$
p_{s+t} \circ U_{s, t}^{Y}=U_{s, t}^{X} \circ\left(p_{s} \otimes p_{t}\right), s, t \in \mathcal{S} .
$$


Embedding a subproduct system as a subsystem of a product system plays an essential role in the dilation theory of CP-semigroups [8]. It is easy to see that every subproduct system $X$ over $\mathbb{N}$ is a subproduct subsystem of the product system $\left\{X(1)^{\otimes n}\right\}_{n \in \mathbb{N}}$. It is harder to show that every subproduct system over $\mathbb{R}_{+}$ is a subsystem of a product system - this has been done in $[4,6,7]$. It seems that the same result for $\mathbb{N}^{2}$ follows from [10]. In [8] we showed that every subproduct system of finite dimensional Hilbert spaces over $\mathbb{N}^{k}$ is a subproduct subsystem of a product system over $\mathbb{N}^{k}$. Can this always be done?

Explicitly, let $\mathcal{S}$ be either $\mathbb{N}^{k}$ or $\mathbb{R}_{+}^{k}$. Is every subproduct system over $\mathcal{S}$ a subproduct subsystem of a product system? A positive solution will immediately lead to new dilation theorems for CP-semigroups over $\mathbb{N}^{k}$ and $\mathbb{R}_{+}^{k}$. A negative answer will be fantastically interesting.

\section{REFERENCES}

[1] Wm. B. Arveson, Continuous analogues of Fock space, Mem. Amer. Math. Soc., No. 409, American Mathematical Society, 1989.

[2] Wm. B. Arveson, The index of a quantum dynamical semigroup, J. Funct. Anal., Vol. 146, No. 2 (1997), 557588.

[3] Wm.B. Arveson, Non commutative dynamics and E-semigroups, Springer Monographs in Math., Springer-Verlag, 2003.

[4] B. V. R. Bhat and M. Skeide, Tensor product systems of Hilbert modules and dilations of completely positive semigroups, Infinite Dimensional Analysis, Quantum Probability and Related Topics, Vol. 3, (2000), 519-575.

[5] N. J. Fowler, Discrete product systems of Hilbert bimodules, Pac. J. Math., Vol. 204, No. 2 (2002), 335-375.

[6] D. Markiewicz, On the product system of a completely positive semigroup, J. Funct. Anal.. Vol. 200, No. 1 (2003), 237-280.

[7] P. Muhly and B. Solel, Quantum Markov Processes (Correspondences and Dilations), Internat. J. Math. Vol. 13, No. 8 (2002), 863-906.

[8] O. M. Shalit and B. Solel, Subproduct systems, preprint, arXiv:0901.1422v1 [math.OA].

[9] M. Skeide, Product Systems; a Survey with Commutants in View, to appear in the proceedings of the 2006 Quantum Probability Conference in Nottingham.

[10] B. Solel, Representations of product systems over semigroups and dilations of commuting CP maps, J. Funct. Anal., Vol. 235, No. 2 (2006) 593-618.

\section{Michael Skeide: Some Problems}

On the Universality of the Module Approach. Let $T$ be a Markov semigroup on a unital $C^{*}$ or von Neumann algebra $\mathcal{B}$. Suppose we have a general dilation of $T$ to an $E_{0}$ or automorphism semigroup $\vartheta$ on a $C^{*}$ or von Neumann algebra $\mathcal{A}$. In the introduction we proposed to pass, via GNS-construction for the expectation $\mathfrak{p}: \mathcal{B} \rightarrow \mathcal{A}$, to a dilation where the semigroup $\vartheta$ acts on a $\mathcal{B}^{a}(E)$ and where $\mathfrak{p}=\langle\xi, \bullet \xi\rangle$ for some unit vector $\xi \in E$. 

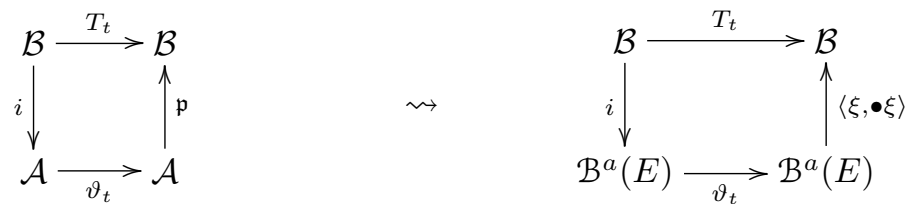

To do so, it is necessary and sufficient that: Firstly, $\vartheta$ respects the kernel of the GNS-representation of $\mathcal{A}$ on $E$, so that it gives rise to an $E_{0}$-semigroup on the image of $\mathcal{A}$ in $\mathcal{B}^{a}(E)$. Secondly, the $E_{0}$-semigroup on the subset of $\mathcal{B}^{a}(E)$ extends to all of $\mathcal{B}^{a}(E)$.

We conjecture that this is always possible, at least if the algebra $\mathcal{A}$ is not too big. By this we mean, for instance, that $\mathcal{A}$ is generated by the image of $\mathcal{B}$ and its time shifts. (If $\mathcal{A}$ has parts that are essentially unrelated to the dilation, then it should be possible to violate our requirements.) Our conjecture is supported by the observation, formulated very carefully, that there is not a single example of a dilation where it was explicitly known that our conjecture is false.

Our first open problem is: Prove the conjecture or, otherwise, give a counter example. For that is is indispensable to find a "good" formulation for that $\mathcal{A}$ is not "too big".

Suppose we have an automorphic dilation $\vartheta$ (after the first part, acting on $\mathcal{B}^{a}(E)$ ). In all existing cases, the automorphism semigroup $\vartheta$ will be inner, that is, will be implemented by a unitary semigroup in $\mathcal{B}^{a}(E)$. Unfortunately, the product system of an inner automorphism semigroup is the trivial one.

Our second open problem asks if every such inner automorphic dilation (fulfilling suitable minimality conditions) may be restricted to a proper $E_{0}$-semigroup on the "future part" of the algebra $\mathcal{B}^{a}(E)$. More precisely, suppose that $E_{+}=$ $\overline{\text { span }} \vartheta_{\mathbb{R}_{+}} \circ i(\mathcal{B}) \xi \subset E$, so that $\vartheta$ gives rise to an $E_{0}$-semigroup $\vartheta^{\prime}$ on $\overline{\operatorname{alg}} \vartheta_{\mathbb{R}_{+}} \circ i(\mathcal{B}) \subset$ $\mathcal{B}^{a}\left(E_{+}\right)$. Does this $E_{0}$-semigroup extend to all of $\mathcal{B}^{a}\left(E_{+}\right)$? Moreover, does there exist a unital embedding of $\mathcal{B}^{a}\left(E_{+}\right)$into $\mathcal{B}^{a}(E)$ such that the extension of $\vartheta^{\prime}$ may be identified with the restriction of $\vartheta$ to $\mathcal{B}^{a}\left(E_{+}\right) \subset \mathcal{B}^{a}(E)$ ? Note that by the representation theory [MSS06], this means that $E$ factors as $E=E_{+} \odot F$ for some multiplicity bimodule $F$. As a case study, we propose to investigate the two-sided time shift on the $q$-Fock space over the whole axis $\mathbb{R}$.

A positive answer to the first question will show that all dilations maybe discussed conveniently in the language of Hilbert modules. A positive answer to the second question shows that even if we start with an automorphic dilation, then we always find a proper $E_{0}$-semigroup on a subalgebra $\mathcal{B}^{a}\left(E_{+}\right)$. Both together would show that our approach proposed as the main theme of the workshop - discuss dilations in terms of Hilbert modules and classify them by product systems - is universal.

Problems around Spatial Markov Semigroups. A Markov semigroup is spatial, if it dominates and elementary CP-semigroup, that is, if there is a (sufficiently continuous) semigroup $c=\left(c_{t}\right)_{t \in \mathbb{R}_{+}}$of elements in $\mathcal{B}$ such that the difference $T_{t}-c_{t}^{*} \bullet c_{t}$ is CP for all $t$. Spatial Markov semigroup on a $C^{*}$-algebra are 
always uniformly continuous with a Christensen-Evans generator

$$
\mathcal{L}(b)=\langle\zeta, b \zeta\rangle+b \beta+\beta^{*} b,
$$

where $\zeta$ is an element in a Hilbert bimodule $F$ and $\beta \in \mathcal{B}$. Their product systems always embed into a time ordered product system of Fock modules (which is even completely spatial), but they need not coincide; see [BLS08]. Spatial Markov semigroups on von Neumann algebras have a richer structure. Their product systems are always spatial. But only if the semigroup is uniformly continuous we can show that it embeds into and, therefore, coincides with a time ordered product system; see [BBLS04]. There is a huge literature on spatial Markov semigroups on $\mathcal{B}(H)$.

We collect some open questions. Are there nontrivial nonspatial Markov semigroups on $\mathcal{B}(H)$ ? (By nontrivial we mean that they are not nonspatial $E_{0}$-semigroups or just tensor products of such with a spatial Markov semigroup.) Are there spatial Markov semigroups on $\mathcal{B}(H)$ whose product system is non-Fock (unlike quantum Ornstein-Uhlenbeck)? Which nonspatial Markov semigroups on a von Neumann algebra $\mathcal{B} \subset \mathcal{B}(H)$ admit spatial extensions to $\mathcal{B}(H)$ ? (Recall that the product system of the Markov semigroups on $L^{\infty}(\mathbb{R})$ of classical Brownian motion and classical Ornstein-Uhlenbeck processes are all nonspatial, but their quantum extensions to $\mathcal{B}\left(L^{2}(\mathbb{R})\right)$ are even type I.) More generally, if a Markov semigroup $T$ on $\mathcal{B}$ admits an extension to $\mathcal{C} \supset \mathcal{B}$, then the product system of $T$ sits inside the product system of its extension. In how far such an embedding does change the basic classification of the product system?

There is the theory of form generators for Markov semigroups on $\mathcal{B}(H)$; see [CF98]. They are all spatial. Are these all spatial Markov semigroups, or are there other spatial ones? Some of them could be dilated to a symmetric Fock space, others not. Are the latter possibly candidates for Markov semigroups that are not type I? Form generators of Markov semigroups on $\mathcal{B}(H)$ resemble the Lindblad form of the uniformly continuous case. Do generators of spatial Markov semigroups on general von Neumann algebras resemble the Christensen-Evans form [CE79] of bounded generators? Is it possible to adapt the theory of [CF98] to general von Neumann algebras?

$n$-Dimensional Free Flows and Their Units. We mentioned already several open problems about free flows and their free and tensor product systems in our talk; see Page 36. Here we wish to propose a generalization to $n$-parameter free flows. This is closely related to the talks of Shalit (Page 27) and Skalski (Page $39)$.

The idea is the following: Once we have an $n$-parameter $E_{0}$-semigroup $\vartheta$ on $\mathcal{B}^{a}(E)$, one may try to find all (unital) units of its product system. Such a unit gives, then, necessarily rise to an $n$-parameter Markov semigroup. Question: Are these semigroups products of strongly commuting (see Shalit's talk) one-parameter semigroups or not? 
To follow this idea it is, therefore, necessary to construct $n$-parameter $E_{0}$-semigroups as candidates. We propose a general procedure starting from free product systems, and pose the open problem to put the above program into practise.

Suppose, we have $n$ one-parameter free product systems $E^{i^{*}}$. (We described in our talk, how to obtain them starting with $n$ spatial product systems.) Then the free products

$$
E_{\left(t_{1}, \ldots, t_{n}\right)}:=E^{1} * \ldots * E^{n}
$$

form an $n$-parameter free product system. Doing the inductive limit over the reference vector (as discussed for the one-parameter case in our talk), we obtain an $E_{0}$-semigroup (a noise, actually). In the case of the full Fock module over $E=L^{2}\left(\mathbb{R}_{+}^{n}, F\right)$, we obtain the $E_{0}$-semigroup induced by the $n$-dimensional right shift. We know all the units in the one-dimensional case (see [Ske06b]), and it appears natural to adapt the same methods to more parameters. We do not exclude the possibility that (due to the high degree of noncommutativity in free products) there are no units but the trivial one (consisting of the reference vectors). We simply do not know. That is why we pose it as an open problem.

\section{Boris Tsirelson: CAN we get type II ARVeson systems from Lévy PROCESSES?}

The Arveson system of a Lévy process on $\mathbb{R}$ is always of type I, i.e. the units are total. The same holds for Lévy processes with values in Lie groups, since they can be constructed from an equivalent noise in the tangent space, i.e. Lie algebra. A similar result was shown for Brownian motions in the unitary group $\mathcal{U}(H)$ of an infinite-dimensional separable Hilbert $H$, which is continuous in the strong topology, cf. [1]. Lévy processes with values in diffeomorphisms groups also give always rise to type I Arveson systems. On the other hand, it is possible to construct Lévy processes with values in semigroups, which give type II Arveson systems, cf. [2], Sections 2c, 2d, 4d.

It is unknown if this can happen for Lévy processes with values in groups. E.g., do there exist Lévy processes with values in a group of homeomorphisms (e.g. of the circle) whose Arveson system is of type II? This question was asked in 1998, see Conjecture 1.11 in [1], and is still open, see Sect. 8a in [2].

\section{REFERENCES}

[1] Boris Tsirelson, Unitary Brownian motions are linearizable, report MSRI 1998-027, arXiv:math/9806112, 1998.

[2] Boris Tsirelson, Nonclassical stochastic flows and continuous products, Probability Surveys, 1, (2004), 173-298 (electronic), arXiv:math/0402431. 